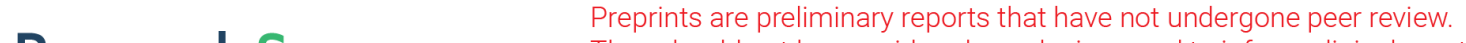 They should not be considered conclusive, used to inform clinical practice, or referenced by the media as validated information. \\ Dynamic Behavior of T-beam Resonator With Repulsive Actuation
}

\section{Yu Tian ( $\nabla$ ytian30@binghamton.edu )}

Binghamton University https://orcid.org/0000-0002-8966-8140

\section{Meysam Daeichin}

Binghamton University

\section{Shahrzad Towfighian}

Binghamton University https://orcid.org/0000-0002-5084-7395

\section{Research Article}

Keywords: MEMS resonators, Repulsive force actuation, Nonlinear dynamics, Primary resonances, Principal parametric resonances

Posted Date: April 20th, 2021

DOl: https://doi.org/10.21203/rs.3.rs-397667/v1

License: @ (i) This work is licensed under a Creative Commons Attribution 4.0 International License. Read Full License

Version of Record: A version of this preprint was published at Nonlinear Dynamics on December 2nd, 2021. See the published version at https://doi.org/10.1007/s11071-021-07073-z. 


\title{
Dynamic Behavior of T-beam Resonator with Repulsive Actuation
}

\author{
Yu Tian - Meysam Daeichin - Shahrzad Towfighian
}

Received: date / Accepted: date

\begin{abstract}
We introduce a MEMS resonator that uses a "T"-shape beam driven by repulsive force, of which the first advantage is to avoid pull-in instability; thus, high enough voltages can be applied to the MEMS system to tune the center frequency. A T-beam model is derived from the beam-paddle hypothesis, and theoretical analysis regarding both static and dynamic behaviors, including primary resonance and secondary resonance, is conducted. This study shows an electrostatic T-beam resonator's feasibility based on repulsive force and outlines its advantages over a traditional cantilever beam resonator. Additional micro-paddle to the micro-beam means larger surface for absorption of targeted analytes and lower natural frequency, but higher resonant responses. We present a thorough analysis of primary and parametric resonances, which can enhance the system signal-to noise ratio and response time. This design enables potential applications in MEMS mass-sensors, where a large area for attachment and a high resolution are often vital.
\end{abstract}

Keywords MEMS resonators · Repulsive force actuation - Nonlinear dynamics · Primary resonances · Principal parametric resonances

Yu Tian

E-mail: ytian30@binghamton.edu

Meysam Daeichin

E-mail: mdaeich1@binghamton.edu

Shahrzad Towfighian (Corresponding author)

Tel.: +607-777-5315

E-mail: stowfigh@binghamton.edu

State University of New York at Binghamton

4400 Vestal Parkway

Binghamton, New York 13902

\section{Introduction}

Capacitive sensing and actuation have been the most common method of transduction in MEMS transducers for the past few decades [1-3]. One could enumerate the compatibility with microfabrication technology, low sensitivity to temperature change, and low power consumption amongst the reasons for the popularity of this transduction scheme [1-3]. The basic design of this transducers has not changed significantly since their introduction in 70s and 80s [4]. They are mainly based on a two-electrode design such as parallel plate or combdrive configurations. These designs suffer from pull-in instability [5], small stroke range [6], and low allowed voltage that hampers the sensitivity of these devices[4].

Repulsive electrode configuration is a multi-electrode capacitive scheme that addresses the issues associated with conventional two-electrode designs $[7,8]$. Since their introduction by Lee et al. [7], this scheme has been explored to realize different MEMS sensors and actuators such as Micro-Mirrors [9,10], microphones [11], accelerometers [12], MEMS switches [13] and resonators [8]. In this study, we investigate the feasibility of using repulsive electrode configuration to build a MEMS gas sensors.

MEMS gas sensors employ a compliant element such as microcantilever beam $[14,15]$. Special coating is used for the microbeam to make it absorb certain molecules in the environment $[16,17]$. When the gas molecules adheres to the microbeam, the microbeam deflects and its resonance frequency changes. Either of these quantities (deflection or resonance frequency) could be measured $[18,19,15]$ to determine the concentration of the target 
gas in the environment.

Static gas sensors measure the deflection of the microstructure with the aid of proper electronic circuitry [18]. Although the operation of the sensor in static mode is easier compared to dynamic mode, the sensitivity of dynamics gas sensors is usually higher [14]. Dynamic gas sensors measure the shift in the resonance frequency of the microstructure that is caused by the added mass of the gas molecules $[14,20]$. To amplify the shift in the resonance frequency, the sensor is designed with a large surface area that is in contact with the gas. Larger surface area makes it possible for more molecules to adhere to the microstructure and therefore more significant change in the mass and resonance frequency. One of the common method to increase the surface area is to attach a paddle to the microstructure $[14,21]$.

In the present paper, we study the statics and dynamics of a gas sensor that employs repulsive electrode configuration. Figure 1 shows the schematic of a gas sensor with a paddle at the free end of the microcantilever beam. The electrode configuration is consisted of three separate electrodes. The first electrode is the beam and the paddle which is referred to as the moving electrode. The second electrode is placed underneath the beam which is called the bottom electrode. The third electrode is a continuous side electrode that is placed on the substrate within a certain distance around the perimeter of the microstructure as shown in Figure 1. Applying voltages on the side electrode while making the moving and bottom electrodes grounded, creates an electrostatic force on the moving electrode. This force is upward, meaning that it pushes the microstructure away from the bottom electrode $[8,22]$. This nature of the electrostatic force eliminates the pull-in possibility between the moving and bottom electrodes $[11,23]$. Because the beam goes further away from the bottom electrode when a DC voltage is applied on the side electrode, the effective gap between the moving and bottom electrodes increases [8]. This provided more room for the vibration of the microstructure enabling it to have large stroke motion $[8,10,22]$.

The organization of the paper is as follows. Section 2 presents the derivation of the governing equation for a micro-cantilever with paddle that is subjected to repulsive electrode configuration. A lumped parameter model is extracted from the continuous governing equation of motion using Galerkin's decomposition method. The discretized equation resembles the nonlinear Mathieu's equation that are solved using the

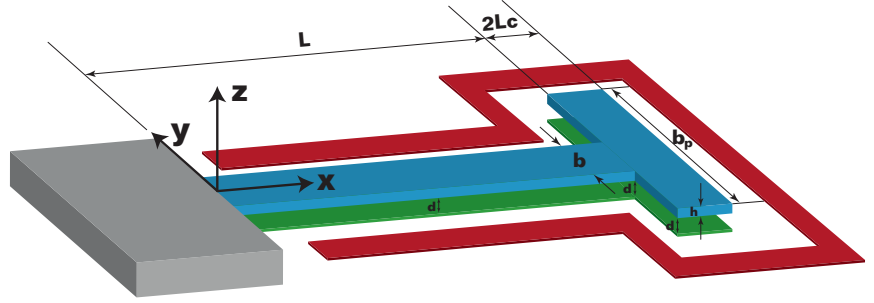

Fig. 1: Schematic of the T-beam repulsive force actuator assembly.

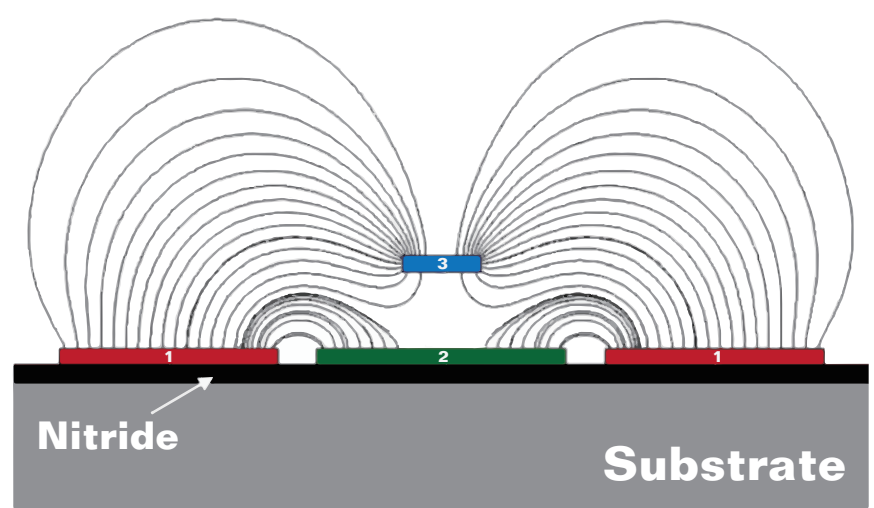

Fig. 2: Repulsive force electrode configuration with Tbeam (3), fixed center electrode (2), and fixed side electrodes (1).

method of multiple scales. In section 3, the static response of the microbeam to DC voltage excitation is obtained numerically with MATLAB. The dynamic response of the microstructure to $\mathrm{DC}+\mathrm{AC}$ voltage excitation is investigated as well. The primary resonance and principal parametric resonance of the microstructure are analyzed and discussed in this section. Finally, section 4 concludes the paper.

\section{Problem Formulation}

The T-beam consists of two coupled beams: a primary micro-beam fixed at one end with another shorter secondary micro-beam attached at the tip. For reasonable simplification when doing both static and dynamic analysis, here we treat the structure as an Euler-Bernoulli beam (primary beam) of width $b$, thickness $h$ with a micro-paddle (secondary beam) attached at the end, which is a rigid body with mass $\hat{M}$, mass moment of inertia $J=\frac{1}{3} \hat{M} \hat{L}_{C}^{2}$, and center of mass lying at a distance $\hat{L}_{C}=\hat{x}_{C}-L$ along the center line from the primary beam tip. 


\begin{tabular}{|c|c|c|}
\hline Parameter & Symbol & Value \\
\hline Micro-beam length $(\mu \mathrm{m})$ & $L$ & 500 \\
Micro-paddle length $(\mu \mathrm{m})$ & $2 L_{c}$ & 20 \\
Micro-beam width $(\mu \mathrm{m})$ & $b$ & 20 \\
Micro-paddle width $(\mu \mathrm{m})$ & $b_{p}$ & 200 \\
Beam height $(\mu \mathrm{m})$ & $h$ & 2 \\
Beam-electrode gap $(\mu \mathrm{m})$ & $d$ & 2 \\
Elastic modulus $(\mathrm{GPa})$ & $\mathrm{E}$ & 150 \\
Density $\left(k g / \mathrm{m}^{3}\right)$ & $\rho$ & 2330 \\
Poisson's ratio & $\nu$ & 0.22 \\
Force constant & $p_{9}$ & $-1.025 \times 10^{34}$ \\
Force constant & $p_{8}$ & $2.635 \times 10^{30}$ \\
Force constant & $p_{7}$ & $-2.895 \times 10^{26}$ \\
Force constant & $p_{6}$ & $1.774 \times 10^{22}$ \\
Force constant & $p_{5}$ & $-6.649 \times 10^{17}$ \\
Force constant & $p_{4}$ & $1.576 \times 10^{13}$ \\
Force constant & $p_{3}$ & $-2.387 \times 10^{8}$ \\
Force constant & $p_{2}$ & $2.247 \times 10^{3}$ \\
Force constant & $p_{1}$ & $-8.100 \times 10^{-3}$ \\
Force constant & $p_{0}$ & $-1.043 \times 10^{-7}$ \\
Force constant & $r_{9}$ & $-4.480 \times 10^{33}$ \\
Force constant & $r_{8}$ & $1.186 \times 10^{30}$ \\
Force constant & $r_{7}$ & $-1.346 \times 10^{26}$ \\
Force constant & $r_{6}$ & $8.577 \times 10^{21}$ \\
Force constant & $r_{5}$ & $-3.384 \times 10^{17}$ \\
Force constant & $r_{4}$ & $8.622 \times 10^{12}$ \\
Force constant & $r_{3}$ & $-1.436 \times 10^{8}$ \\
Force constant & $r_{2}$ & $1.478 \times 10^{3}$ \\
Force constant & $r_{1}$ & $-4.772 \times 10^{-3}$ \\
Force constant & $r_{0}$ & $-8.767 \times 10^{-8}$ \\
\hline
\end{tabular}

Table 1: Beam material and geometric properties.

\subsection{Equation of Motion}

We derive the equation of motion for the T-beam resonator system using the extended Hamilton's principle, shown in Equation (1).

$\int_{t_{1}}^{t_{2}}\left(\delta T_{\text {total }}-\delta U_{\text {total }}+\delta W_{N C}\right) d t=0$

where $\delta T$ is the variational kinetic energy, $\delta U$ is the variational potential energy, and $\delta W_{N C}$ is the virtual work of non-conservative forces, such as damping. Due to the beam-paddle hypothesis, the secondary beam is assumed to be a rigid body and thus hardly contributes the potential energy but significantly associates the kinetic energy [1]. Here, the T-beam resonator system is treated as a conservative electromechanical system. The work done by the electrostatic forces on the T-beam is considered conservative and included in the potential energy. The virtual work done by non-conservative external force comes from viscous dissipation, mostly on the primary beam, and the portion on the secondary beam is neglected. The kinetic, potential energy and virtual work for the micro-system are represented as Equations (2)-(9).

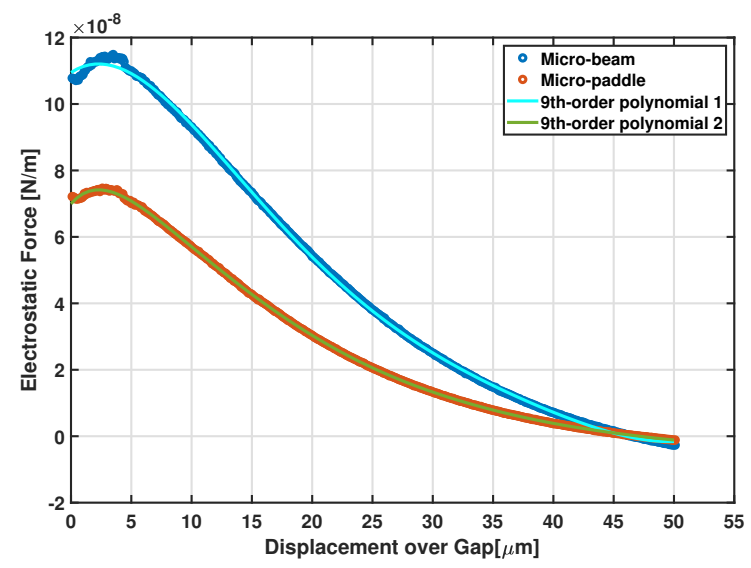

Fig. 3: Electrostatic force profile for T-beam actuator shown in Figure 1. Equilibrium position at 46 microns for dimensions in Table 1.

$T_{\text {prim }}=\int_{0}^{L} \frac{1}{2} \rho A_{P} \hat{W}_{\hat{t}}^{2}(\hat{x}, \hat{t}) d \hat{x}$
$T_{\text {sec }}=\frac{1}{2} \hat{M}\left[\hat{W}_{\hat{t}}(L, \hat{t})+{\hat{L_{C}}}_{\hat{W_{\hat{t}} \hat{x}}}(L, \hat{t})\right]^{2}+\frac{1}{2} J \hat{W}_{\hat{t} \hat{x}}^{2}(L, \hat{t})$

$T_{\text {total }}=T_{\text {prim }}+T_{\text {sec }}$

$U_{\text {prim }}=\int_{0}^{L} \frac{1}{2} E I_{P} \hat{W}_{\hat{x} \hat{x}}^{2}(\hat{x}, \hat{t}) d \hat{x}+\int_{0}^{L} V^{2} f_{1}(d+\hat{W}) \delta \hat{W} d \hat{x}$

$U_{s e c}=\left.\int_{0}^{2 \hat{L_{C}}} V^{2}\left(f_{2}\left(d+\hat{W}+\hat{W}_{\hat{x}} s\right) \delta\left(\hat{W}+\hat{W}_{\hat{x}} s\right)\right)\right|_{\hat{x}=L} d s$

$U_{\text {total }}=U_{\text {prim }}+U_{\text {sec }}$

$\approx \int_{0}^{L} \frac{1}{2} E I_{P} \hat{W}_{\hat{x} \hat{x}}^{2}(\hat{x}, \hat{t}) d \hat{x}+\int_{0}^{L} V^{2}\left(f_{1}(d+\hat{W})\right.$

$\left.+2 \frac{\hat{L_{C}}}{L_{2}}(d+\hat{W}) \delta[\hat{x}-\hat{L}]\right) \delta \hat{W} d \hat{x}$

$\delta W_{N C}=\int_{0}^{L} c \hat{W}_{\hat{t}}(\hat{x}, \hat{t}) \delta \hat{W} d \hat{x}$

The numerical profile for repulsive force is obtained and fit as two 9th-order polynomials from a finite element simulation for the intended fabrication dimensions in COMSOL (Figure 3). It is worthwhile to mention that the repulsive force on the paddle as in Equation (6) is considered approximately as a point force at the tip of the beam by multiplying the force per unit length for the paddle by the length of the paddle $\left(2 \hat{L_{C}}\right)$ and consider the gap at the tip of the primary beam as the gap all across the paddle as in Equation (8). Expressing 


\begin{tabular}{|c|c|}
\hline Parameter & Substitution \\
\hline$x$-direction position & $x=\hat{x} / L$ \\
$W$-direction position & $W=\hat{W} / h$ \\
$t$-time & $t=\hat{t} / T$ \\
$c$-damping & $c=\hat{c} L^{4} / E I T$ \\
Time constant & $T=\sqrt{\rho A L^{4} / E I}$ \\
M-mass of paddle & $M=\hat{M} / \rho A L$ \\
$L_{C}$-length of paddle & $L_{C}=\hat{L_{C} / L}$ \\
Force constant & $\alpha=L^{4} / E I h$ \\
Force constant & $\beta_{1}=2 L_{C}^{2} \alpha$ \\
Force constant & $\beta_{2}=8 L_{C}^{3} h \alpha / 3$ \\
Force constant & $\beta_{3}=2 L_{C} \alpha$ \\
Force constant & $\beta_{4}=2 L_{C}^{2} h \alpha$ \\
\hline
\end{tabular}

Table 2: Nondimensional Substitutions.

and solving for the variation of the energy terms and plugging them into Equation (1), yields the nondimensionalized (with Table 2) governing equation of motion for the system shown as

$$
\begin{aligned}
& \ddot{W}+c \dot{W}+W_{x x x x} \\
& \quad+\alpha V^{2}\left(\sum_{i=0}^{9} p_{i} h^{i} W^{i}+2 L_{C} \delta[x-1] \sum_{i=0}^{9} r_{i} h^{i} W^{i}\right)=0
\end{aligned}
$$

where $W$ is the z-direction beam displacement. Boundary conditions are accordingly shown in Equations (11) for $x=0$ and Equation (12)-(13) for $x=1$,

$W=0, W_{x}=0$

$W_{x x}+M L_{C} \ddot{W}+\left(M L_{C}^{2}+\frac{M\left(2 L_{C}\right)^{2}}{12}\right) \ddot{W}_{x}=0$

$W_{x x x}-M \ddot{W}-L_{C} M \ddot{W}_{x}=0$

\subsection{Mechanical Mode Shapes}

Determining the microstructure's natural frequencies as accurately as possible is crucial for characterizing their actual geometry and boundary conditions, revealing their operating range and restrictions, and enabling accurate calibration of resonator-based devices [24]. Set damping term $c=0$ and forcing terms $V=0$, linear eigenvalue problem is obtained:

$$
\begin{aligned}
& \ddot{W}+W_{x x x x}=0 \quad(0 \leq x \leq 1) \\
& W_{x x}+M L_{C} \ddot{W}+\frac{4}{3} L_{C}^{2} M \ddot{W}_{x}=0 \quad(x=1) \\
& W_{x x x}-M \ddot{W}-L_{C} M \ddot{W}_{x}=0 \quad(x=1) \\
& W=0 \quad(x=0) \\
& W_{x}=0 \quad(x=0)
\end{aligned}
$$

\begin{tabular}{|c|c|c|c|}
\hline Mode & $\beta$ & frequency $(\mathrm{kHz})$ & $c_{2} / c_{1}$ \\
\hline 1 & 1.4590 & 6.2779 & -0.7776 \\
2 & 4.0701 & 48.854 & -1.0110 \\
3 & 7.0685 & 147.35 & -0.9997 \\
4 & 10.0879 & 300.12 & -1.000 \\
\hline
\end{tabular}

Table 3: Nondimensional Substitutions.

Using separation of variables $W(x, t)=\phi(x) q(t)$ and setting $\ddot{q}(t)=-\omega^{2} u(t)$, where $\omega$ stands for non-dimensional natural frequency, the mode shape is written as

$\phi(x)=c_{1} \cos (\beta x)+c_{2} \sin (\beta x)+c_{3} \cosh (\beta x)+c_{4} \sinh (\beta x)$

where $\beta$ is also a non-dimensional parameter defined by $\omega=\beta^{2}$. Combining boundary conditions Equations (15)-(18), we can find $c_{3}=-c_{1}$ and $c_{4}=-c_{2}$, and the characteristic equation is described as

$\left[\begin{array}{ll}A & B \\ C & D\end{array}\right]\left[\begin{array}{l}c_{1} \\ c_{2}\end{array}\right]=\left[\begin{array}{l}0 \\ 0\end{array}\right]$

where

$$
\begin{aligned}
A= & \beta^{2}(-\cos (\beta)-\cosh (\beta))-M L_{C} \omega^{2}(\cos (\beta)-\cosh (\beta)) \\
& -\frac{4}{3} L_{C}^{2} M \omega^{2} \beta(-\sin (\beta)-\sinh (\beta)) \\
B= & \beta^{2}(-\sin (\beta)-\sinh (\beta))-M L_{C} \omega^{2}(\sin (\beta)-\sinh (\beta)) \\
& -\frac{4}{3} L_{C}^{2} M \omega^{2} \beta(\cos (\beta)-\cosh (\beta)) \\
C= & \beta^{3}(\sin (\beta)-\sinh (\beta))+M \omega^{2}(\cos (\beta)-\cosh (\beta)) \\
& +M L_{C} \omega^{2} \beta(-\sin (\beta)-\sinh (\beta)) \\
D= & \beta^{3}(-\cos (\beta)-\cosh (\beta))+M \omega^{2}(\sin (\beta)-\sinh (\beta)) \\
& +M L_{C} \omega^{2} \beta(\cos (\beta)-\cosh (\beta))
\end{aligned}
$$

For eigenvalues and mechanical mode shapes, we need to obtain the non-trivial solutions to Equation (20). Solving $\left|\begin{array}{ll}A & B \\ C & D\end{array}\right|=0$ with MATLAB, values of $\beta$ and $c_{2} / c_{1}$ for the first three mechanical modes are shown in Table 3 .

\subsection{Reduced Order Model}

Once the mechanical modes shapes $\phi_{m}{ }^{\prime} s$ is known, with Galerkin's method, Equation (1) is reduced from PDE to a set of ordinary differential equations (ODEs), as Equation (21), that can be solved numerically. 


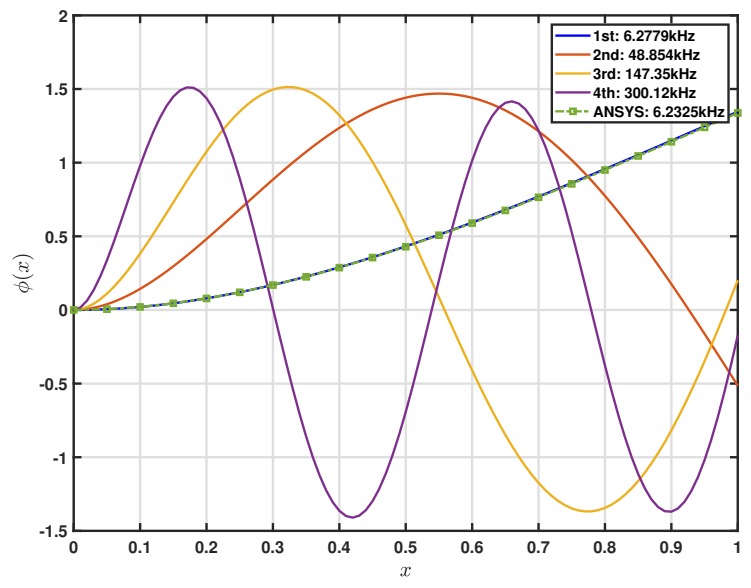

Fig. 4: First four mode shapes from Matlab and first mode shape from ANSYS.

$\int_{0}^{1}\left(\phi_{m}(x)\right)\left(\sum_{j=1}^{N} \phi_{j}(x) \ddot{q}_{j}(t)\right.$

$\left.\left.+c \sum_{j=1}^{N} \phi_{j}(x) \dot{q}_{j}(t)\right) d x+\sum_{j=1}^{N} \phi_{j}^{\prime \prime \prime \prime}(x) q_{j}(t)\right) d x$

$+\alpha V^{2} \sum_{i=0}^{9} p_{i} h^{i} \int_{0}^{1}\left(\phi_{m}(x)\right) \sum_{j=1}^{N}\left(\phi_{j}(x) q_{j}(t)\right)^{i} d x$

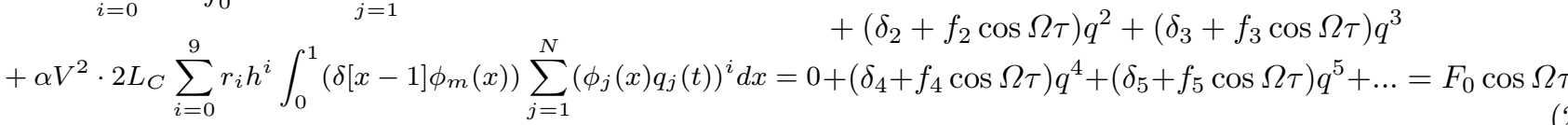

where $m=1,2, \ldots, N$ and $\mathrm{N}$ represents the number of mode shapes in consideration.

For a one-mode approximation, Equation (10) is reduced to,

$$
\begin{aligned}
& \int_{0}^{1}\left(\phi_{1}(x)\right)^{2} d x \ddot{q}_{1}(t)+c \int_{0}^{1}\left(\phi_{1}(x)\right)^{2} d x \dot{q}_{1}(t) \\
& +\int_{0}^{1} \phi_{1}(x) \phi_{1}^{\prime \prime \prime \prime}(x) d x q_{1}(t) \\
& +\alpha V^{2} \sum_{i=0}^{9} p_{i} h^{i} \int_{0}^{1}\left(\phi_{1}(x)\right)^{i+1}\left(q_{1}(t)\right)^{i} d x \\
& +\alpha V^{2} \cdot 2 L_{C} \sum_{i=0}^{9} r_{i} h^{i}\left(\phi_{1}(1)\right)^{i+1}\left(q_{1}(t)\right)^{i}=0
\end{aligned}
$$

\subsection{Dynamic Mathematical Model}

T-beam structure as a sensor or resonator is mostly utilized when resonating. The actuator voltage $V$ is the input signal, a sinusoidal function $V_{A C}$ superimposed on the $V_{D C}$ that is applied to the structure. The terms of force on the paddle from $\delta[x-1]$ are dropped because they are considerably small, around $2 \%$ comparing them on the primary beam, and when applying the combination of two voltages,

$$
\begin{aligned}
V^{2} & =\left(V_{D C}+V_{A C} \cos \omega t\right)^{2} \\
& =V_{D C}^{2}+2 V_{D C} V_{A C} \cos \omega t+\frac{(1+\cos 2 \omega t) V_{A C}^{2}}{2}
\end{aligned}
$$

the term including $V_{A C}^{2}$ is dropped as well since the AC voltage is typically much smaller than DC voltage. Our efforts are then mostly focused on first-order resonance of the driven frequency, which accounts for the total displacement as

$$
q_{\text {total }}(t)=q_{0}+q(t)
$$

where $q_{0}$ stands for the static deflection and $q(t)$ represents the dynamic displacement. Our particular concerns are the dynamic response $q(t)$ about the static equilibrium position shown in Figure 5. Neglecting the squeeze-film damping as well as contact, a generalized form of forced Mathieu equation of motion is formulated as Equation (24) based on one mode approximation,

$$
\begin{aligned}
& \frac{\partial^{2} q}{\partial \tau^{2}}+2 \zeta \frac{\partial q}{\partial \tau}+\left(\omega_{n}^{2}+f_{1} \cos \Omega \tau\right) q \\
& +\left(\delta_{2}+f_{2} \cos \Omega \tau\right) q^{2}+\left(\delta_{3}+f_{3} \cos \Omega \tau\right) q^{3}
\end{aligned}
$$

where

$$
\tau=\omega_{0} t, \Omega=\frac{\omega}{\omega_{0}}, \zeta=\frac{c}{2 \omega_{0}}, \omega_{0}=\sqrt{\frac{K_{11}}{M_{11}}}, \omega_{n}=\sqrt{1+\delta_{1}}
$$

with $\delta_{j}^{\prime} s$ and $f_{j}^{\prime} s$ resulted from DC voltage terms as described

$$
\begin{aligned}
& M_{11}=\int_{0}^{1}\left(\phi_{1}(x)\right)^{2} d x \\
& K_{11}=\int_{0}^{1} \phi_{1}(x) \phi_{1}^{\prime \prime \prime \prime}(x) d x \\
& \delta_{j}=\frac{\alpha V_{D C}^{2}}{K_{11}} \sum_{i=j}^{9}\left(\begin{array}{c}
i \\
i-j
\end{array}\right) p_{i} h^{i} q_{0}^{i-j} \int_{0}^{1}\left(\phi_{1}(x)\right)^{i+1} d x \\
& F_{0}=\frac{-2 \alpha V_{D C} V_{A C}}{K_{11}} \sum_{i=0}^{9} p_{i} h^{i} q_{0}^{i} \int_{0}^{1}\left(\phi_{1}(x)\right)^{i+1} d x \\
& f_{j}=\frac{2 \alpha V_{D C} V_{A C}}{K_{11}} \sum_{i=j}^{9}\left(\begin{array}{c}
i \\
i-j
\end{array}\right) p_{i} h^{i} q_{0}^{i-j} \int_{0}^{1}\left(\phi_{1}(x)\right)^{i+1} d x
\end{aligned}
$$

where $\left(\begin{array}{l}n \\ r\end{array}\right)=\frac{n !}{(n-r) ! r !}$. Before we apply the perturbation method, it's fundamental to keep linear terms while 
making nonlinear terms reasonably small. Here, the book- Inserting Equation (33) and (29) into Equation (28) keeping parameter $\epsilon$ is assumed to be at the order $O\left(10^{-2}\right)$. and (29), eliminating the secular terms and considering The solved steady-state amplitude $q_{0}$, assumed of order $O(1)$, from Equation (22) helps predict and decide the initial order of all the magnitude order of terms in Equation (24), which are listed as in Table 4. Based on theory of multi-scales in perturbation at most to the second-order $\left(\epsilon^{2}\right.$ or $\left.T_{2}\right)$, the terms higher than cubic order have really minimal effect on dynamic behavior, and therefore, we exclude the higher orders and keep dynamic related terms up to cubic terms. Equation (24) is reduced to,

$$
\begin{gathered}
\ddot{q}+2 \zeta \dot{q} \epsilon^{2}+\omega_{n}^{2} q+\epsilon^{2}\left(f_{2} q^{2} \cos \Omega \tau+\delta_{2} q^{2}+\right. \\
\left.\delta_{3} q^{3}+\delta_{4} q^{4}\right)=\epsilon^{2} F_{0} \cos \Omega \tau
\end{gathered}
$$

We then seek an approximate asymptotic solution to Equation (25) by letting

$$
\begin{aligned}
q(\tau)= & q_{0}\left(T_{0}, T_{1}, T_{2}\right)+\epsilon q_{1}\left(T_{0}, T_{1}, T_{2}\right) \\
& +\epsilon^{2} q_{2}\left(T_{0}, T_{1}, T_{2}\right)+O\left(\epsilon^{3}\right)
\end{aligned}
$$

where $T_{0}=\tau, T_{1}=\epsilon \tau$ and $T_{2}=\epsilon^{2} \tau$ represent fast, slow and very slow time scale terms respectively. For achieving solution for Equation (25), it's essential to evaluate the conditions for primary and secondary resonances separately.

\subsubsection{Primary Resonance}

Since the excitation $F_{0}$ is $O\left(\epsilon^{2}\right), \Omega-\omega_{n}$ is assumed to be $O\left(\epsilon^{2}\right)$ for consistency. Hence we introduce $\sigma$ as detuning parameter examining the closeness of $\Omega$ to 1 by putting

$$
\Omega=\omega_{n}+\epsilon^{2} \sigma
$$

To balance the effect of primary-resonance excitation, we need to reorder the equations so that the order of parametric and primary force and $\zeta$ all appears in the $\epsilon^{2}$ order. Substituting Equation (26) and (28) into (25) and equating the coefficients of $\epsilon^{0}, \epsilon^{1}$ and $\epsilon^{2}$ on both sides, we obtain

$\epsilon^{0}: D_{0}^{2} q_{0}+\omega_{n}^{2} q_{0}=0$

$\epsilon^{1}: D_{0}^{2} q_{1}+2 D_{0} D_{1} q_{0}+\omega_{n}^{2} q_{1}=0$

$\epsilon^{2}: D_{0}^{2} q_{2}+2 D_{0} D_{1} q_{1}+\left(D_{1}^{2}+2 D_{0} D_{2}\right) q_{0}$

$+2 \zeta D_{0} q_{0}+\omega_{n}^{2} q_{2}+\delta_{2} q_{0}^{2}+f_{2} q_{0}^{2} \cos \Omega \tau$

$+\delta_{3} q_{0}^{3}+\delta_{4} q_{0}^{4}=F_{0} \cos \Omega \tau$

where $D_{i}=\frac{\partial}{\partial T_{i}}$ The general solution to Equation (24) can be written in form

$q_{0}=A\left(T_{1}, T_{2}\right) e^{i \omega_{n} T_{0}}+\bar{A}\left(T_{1}, T_{2}\right) e^{-i \omega_{n} T_{0}}$ the solubility for the outcome implies that

$$
\begin{array}{r}
e^{i \omega_{n} T_{0}}\left(\frac{f_{2}}{2} A^{2} e^{-i \sigma T_{2}}+\left(f_{2} A \bar{A}-\frac{F_{0}}{2}\right) e^{i \sigma T_{2}}\right. \\
\left.+3 \delta_{3} A^{2} \bar{A}+2 i \omega_{n}\left(\zeta A+D_{2} A\right)\right)=0
\end{array}
$$

Implementing polar notation of $A=\frac{1}{2} a e^{i \beta}$ in terms of amplitude $a$ and nonlinear frequency $\beta$, introducing variable $\gamma=-\beta+\sigma T_{2}$ and separating real and imaginary parts gives derivatives with respect to $T_{2}$ as

$\gamma^{\prime}=\frac{\left(-3 f_{2} a^{2}+4 F_{0}\right) \cos \gamma-3 \delta_{3} a^{3}+8 a \omega_{n} \sigma}{8 a \omega_{n}}$

$a^{\prime}=\frac{\left(f_{2} a^{2}+4 F_{0}\right) \sin \gamma-8 a \omega_{n} \zeta}{8 \omega_{n}}$

The analytical response for primary resonance is therefore represented as

$q=a \cos \left(\beta+\omega_{n} \tau\right)$

By setting right hand side of Equation (31)-(32) for the asymptotic approximation, the amplitude $a$ in terms of detuning parameter is determined by

$\left(\lambda_{1} \lambda_{2}\right)^{2}=\left(\lambda_{3} \lambda_{2}\right)^{2}+\left(\lambda_{4} \lambda_{1}\right)^{2}$

in which

$\lambda_{1}=4 F_{0}-3 f_{2} a^{2}$

$\lambda_{2}=4 F_{0}-f_{2} a^{2}$

$\lambda_{3}=8 a \sigma \omega_{n}-3 \delta_{3} a^{3}$

$\lambda_{4}=-8 a \omega_{m} \zeta$

\subsubsection{Principal Parametric Resonance}

Here, the frequency of driven force is close to the systems natural frequency as

$\Omega=2 \omega_{n}+\epsilon^{2} \sigma$

According to the reordering of $\epsilon^{0}, \epsilon^{1}$ and $\epsilon^{2}$ in Equation (25), we obtain

$$
\begin{aligned}
\epsilon^{0} & : D_{0}^{2} q_{0}+\omega_{n}^{2} q_{0}=F_{0} \cos \Omega T_{0} \\
\epsilon^{1}: & D_{0}^{2} q_{1}+2 D_{0} D_{1} q_{0}+\omega_{n}^{2} q_{1}=0 \\
\epsilon^{2}: & D_{0}^{2} q_{2}+2 D_{0} D_{2} q_{0}+2 \zeta D_{0} q_{0}+\omega_{n}^{2} q_{2} \\
\quad & +\delta_{2} q_{0}^{2}+f_{2} q_{0}^{2} \cos \Omega \tau+\delta_{3} q_{0}^{3}+\delta_{4} q_{0}^{4}=0
\end{aligned}
$$

The general solution to Equation (36) can be represented accordingly

$$
\begin{aligned}
q_{0}=A\left(T_{1}, T_{2}\right) e^{i \omega_{n} T_{0}}+\bar{A}\left(T_{1}, T_{2}\right) e^{-i \omega_{n} T_{0}} & \\
& +\Lambda\left(e^{i \Omega T_{0}}+e^{-i \Omega T_{0}}\right)
\end{aligned}
$$




\begin{tabular}{|c|c|c|c|c|c|c|c|c|c|c|}
\hline$\zeta \dot{q}$ & $\left(1+\delta_{1}\right) q$ & $f_{1} q$ & $\delta_{2} q^{2}$ & $f_{2} q^{2}$ & $\delta_{3} q^{3}$ & $f_{3} q^{3}$ & $\delta_{4} q^{4}$ & $f_{4} q^{4}$ & $\delta_{5} q^{5}$ & $F_{0}$ \\
\hline$O\left(10^{-3}\right)$ & $O(1)$ & $O\left(10^{-5}\right)$ & $O\left(10^{-3}\right)$ & $O\left(10^{-4}\right)$ & $O\left(10^{-3}\right)$ & $O\left(10^{-5}\right)$ & $O\left(10^{-4}\right)$ & $O\left(10^{-6}\right)$ & $O\left(10^{-5}\right)$ & $O\left(10^{-3}\right)$ \\
\hline
\end{tabular}

Table 4: The assumed order of magnitude for the terms shown in Equation (24).

wherein $\Lambda=\frac{F_{0}}{2\left(\omega_{n}^{2}-\Omega^{2}\right)}$. Eliminating the secular terms and considering the solubility for the outcome implies that

$$
\begin{aligned}
& e^{i \omega_{n} T_{0}}\left(2 i \omega_{n}\left(\zeta A+D_{2} A\right)+2 \delta_{2} \Lambda \bar{A} e^{i \sigma T_{2}}\right. \\
& +2 f_{2} A \Lambda+\delta_{3}\left(3 A^{2} \bar{A}+6 A \Lambda^{2}\right) \\
& \left.+\delta_{4}\left(4 A^{3} \Lambda e^{-i \sigma T_{2}}+12 \Lambda^{3} \bar{A} e^{i \sigma T_{2}}+12 A \bar{A}^{2} \Lambda e^{i \sigma T_{2}}\right)\right)=0
\end{aligned}
$$

Then via the notation of $A$ in terms of $a$ and $\beta$ as stated before, we can find the amplitude $a$ of the analytical solution to Equation (23) from Equation (34) as well, in which

$\lambda_{1}=-\left(\frac{9}{2} f_{3} \Lambda^{2}+\frac{a^{2}}{2} f_{3}+\frac{1}{2} f_{1}+2 \delta_{2} \Lambda\right)$

$\lambda_{2}=-\left(\frac{9 a}{4} f_{3} \Lambda^{2}+\frac{a^{3}}{8} f_{3}+\frac{a}{4} f_{1}+\delta_{2} \Lambda a\right)$

$\lambda_{3}=-\left(2 f_{2} \Lambda+6 \Lambda^{2} \delta_{3}+\frac{3 a^{2}}{4} \delta_{3}-\omega_{n} \sigma\right)$

$\lambda_{4}=-\zeta a \omega_{n}$

And the analytical response for principal parametric resonance is

$q=a \cos \left(\beta+\omega_{n} \tau\right)+\frac{F_{0}}{\omega_{n}^{2}-\Omega^{2}} \cos \Omega \tau$

\section{Results and Discussion}

The static and dynamic behaviors or T-beam resonator under repulsive force is studied for a one-mode approximation model. The damping is estimated by

$c=\frac{\beta_{1}^{2}}{Q}$

\subsection{Static Analysis}

For the static analysis, the sensor voltage, $V_{s}$, is a constant DC voltage upwards of $200 \mathrm{~V}$. First, the boundary value problem is derived based on Equation (5) after removing time-dependent components, which results in governing equation as Equation (45):

$W_{s}^{\prime \prime \prime \prime}+\alpha V_{D C}^{2} \sum_{i=0}^{9} p_{i} h^{i} W_{s}^{i}=0$

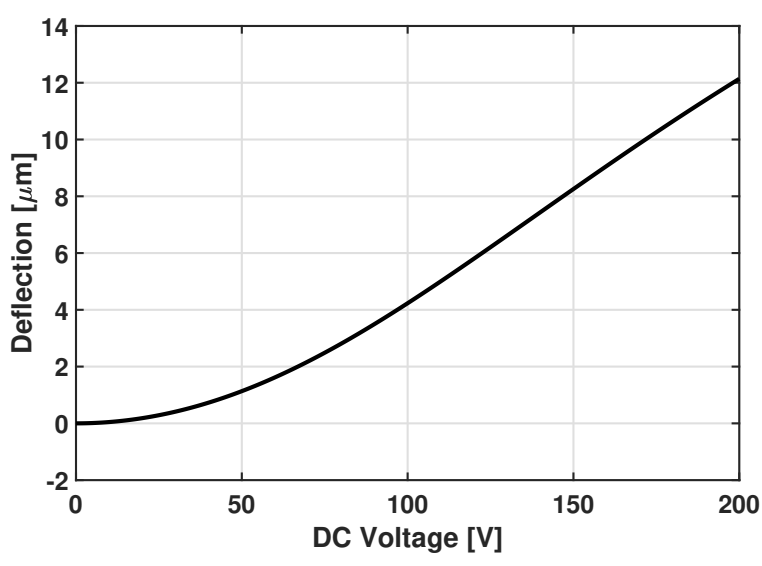

Fig. 5: Static equilibrium position of the end of primary beam as the DC voltage varies.

and the corresponding boundary conditions for $x=0$ and $x=1$ as Equations (46)-(48):

$W=1, W^{\prime}=0$

$W^{\prime \prime}+V^{2}\left(\beta_{1} \sum_{i=0}^{9} r_{i} h^{i} W^{i}+\beta_{2} W^{\prime} \sum_{i=0}^{8}(i+1) r_{i+1} h^{i} W^{i}\right)=0$

$W^{\prime \prime \prime}-V^{2}\left(\beta_{3} \sum_{i=0}^{9} r_{i} h^{i} W^{i}+\beta_{4} W_{x} \sum_{i=0}^{8}(i+1) r_{i+1} h^{i} W^{i}\right)=0$

Noted that the first-order Taylor expansions, $f_{2}(W+$ $\left.W_{x} s\right) \approx f_{2}(W)+f_{2}^{\prime}(W) W_{x} s$, are used when it comes to the nonlinear force on the paddle.

The closed-form $W_{s}=W_{s}(x)$ to represent the static deflection is obtained using bvp4c in Matlab after rewriting it to be four first-order ODEs. The solution $W_{s}(x)$ gives the static $\mathrm{T}$-beam profile $(0 \leq x \leq 1)$ for varying DC voltages shown in Figure 5.

The Jacobian of Equation (22) is computed at the static equilibrium points, and the corresponding eigenvalues of the Jacobian yield the natural frequencies using a one-mode approximation at many voltages $V_{s}$ in Figure 6. Previous research revealed that electrostatic levitation could actuate the cantilever beam to considerable oscillations over an order of magnitude larger than the gap $[25,26]$. T-beam resonator driven by repulsive force shows relatively small natural frequency while produces a high oscillation. Merging T-beam and 


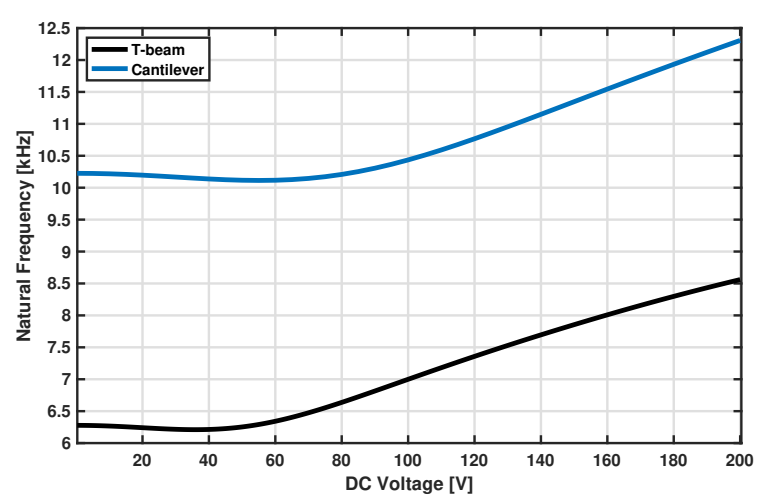

Fig. 6: First natural frequency of the beam as the DC voltage varies.

electrostatic levitation as one single MEMS mass sensor, therefore, enables high resolution in detecting the change in the resonant frequency and less susceptible to noise. It can also motivate the new generation of mass sensors using the phenomenon of mode localization [24] by connecting two single resonators with a coupling beam. Meanwhile, the T-beam undoubtedly enlarges the surface helps detect and measure the presence of different kinds of analytes, including biological species like viruses or bacteria, whether in liquid or gaseous media or specific gases, especially volatile organic compounds (VOCs).

\subsection{Perturbation Analysis}

The Method of multiple scales (MMS) perturbation technique is applied to perturb the micro-actuary near its fundamental primary resonance and, twice of that, to numerically solve the governing equations accordingly and study its primary and principal parametric resonant behaviors. With $\sigma$ serving as detuning parameter and the non-dimensional amplitude $a$, we can investigate more than dynamic T-beam responses when bringing them in of the cantilever of exact dimensions under the same conditions. For determining the steadystate response amplitudes in the vicinity of the principal parametric resonance, the stability of periodic motions is examined by determining the relevant eigenvalues of the Jacobian matrix in the vicinity of the periodic motions. A positive eigenvalue represents an unstable limit circle in phase portrait, and even slight disturbance leads to boundless increasing amplitudes. This effect does not happen if the eigenvalue is negative, which helps the disturbance decay with time. In primary resonance, stable amplitude $a$ represents stable displacement which can be achieved stably, while unsta- ble amplitudes are hard to hold. For principal parametric resonance, things get much intriguing. When amplitude $a$ in our solution, Equation (43), is associated with a negative eigenvalue, the response is defined as principal parametric resonance because the response period is the same as the excitation period. However, when the eigenvalue has a positive sign, it indicates so-called subharmonic resonance. Parametric resonance has a much larger amplitude than subharmonic resonance, which is why most researchers on MEMS resonators are concerning. In order to study the advantages of utilizing Tshaped beam instead of traditionally applied cantilever beam, which is highly popular and widely integrated into MEMS sensing due to the well-known excellent properties, in designing the resonator under repulsive configuration, we can compare T-beam's dynamic behaviors with cantilever beam of same dimensions, meaning 500 microns in length and 20 microns in width. Then a great potential to improve new generations of sensors and actuators by combining T-beam and electrostatic levitation is given.

\subsubsection{Primary Resonance}

Comparing with results of a cantilever beam of the same length in primary resonance as Figure 7, we conclude that the dynamic response of T-beam also shows hysteresis and softening behavior, which is because of a quadratic term and a negative cubic term of the electrostatic force from the forcing profile polynomial fit. These two terms are dominant in the forcing function and explain why the softening is significant even at $\mathrm{AC}$ voltage near $1 \mathrm{~V}$. Meanwhile, less deviation in softening behavior but higher response provides T-beam better resolution when external frequencies vary in the vicinity of primary natural frequency. That means, as the frequency approaches the natural frequency, the oscillation amplitude increases significantly, and when the frequency drops below the resonant frequency, the system experiences hysteresis and drops to a significantly smaller oscillation amplitude.

\subsubsection{Principal Parametric Resonance}

In general, the parametric excitation actuation can be more efficient, requiring less power than the primary resonance excitation, which is highly represented from Figure 7 to Figure 8 when AC voltage keeps the same and DC voltage reduced significantly. Exciting with parametric resonance makes the response faster as the excitation frequency is twice the resonance. Figure 8 shows that the T-beam's parametric resonance achieves a much higher amplitude than the cantilever. Large enough amplitude protects the structure from any dynamic pull-in 


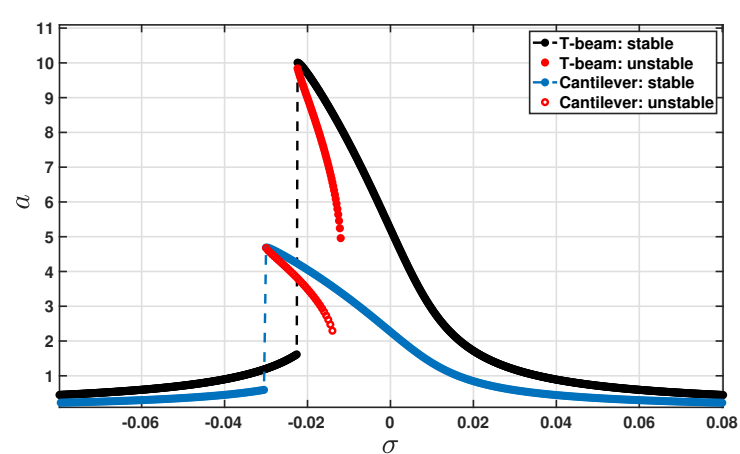

Fig. 7: Comparison of primary resonant response at $V_{D C}=195 \mathrm{~V}$ and $V_{A C}=1 \mathrm{~V}$ for T-beam and cantilever. Quality factor is set to 130 .

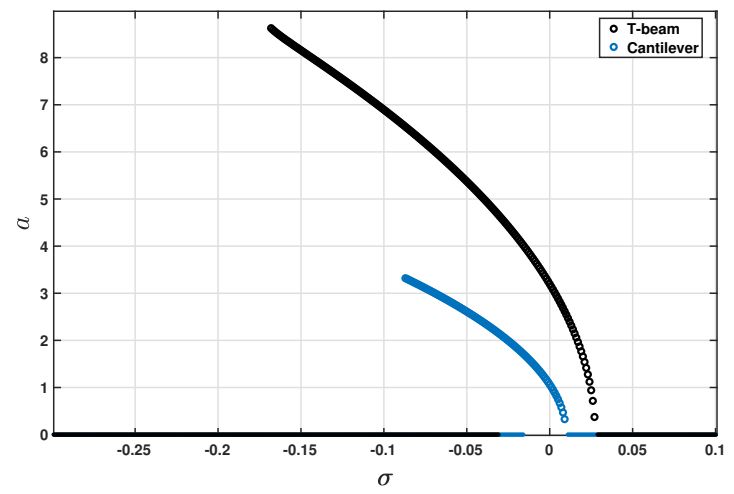

Fig. 8: Comparison of principal parametric resonant response at $V_{D C}=75 \mathrm{~V}$ and $V_{A C}=20 \mathrm{~V}$ for T-beam and cantilever. Quality factor is set to 500 .

instability due to the electrostatic configuration while it results in the collision to the substrate. When the beam moves much close to the electrode, the squeeze film damping effect gets huge [26], which prevents any strikes, which is not accounted for in our model here.

The T-beam resonator can achieve very large dynamic amplitudes from parametric resonance as shown in Figure 9, 10 and 11. If the AC voltage is high enough, the unstable branch, indicated in red, intersects the stable branch, indicated in blue, green and yellow. However, an AC voltage of at least $4.7 \mathrm{~V}$ is necessary to overcome the threshold voltage for parametric resonance when the DC voltage is $75 \mathrm{~V}$ and the quality factor is 500. Since the perturbation method is computationally fast especially in vicinity of targeted frequency range and can predict both stable and unstable branches, it can also be applied to give the principal parametric instability tongue shown as in Figure 12. The unstable area (grey area) is where the parametric resonance happens. This instability tongue shows a minimum thresh-

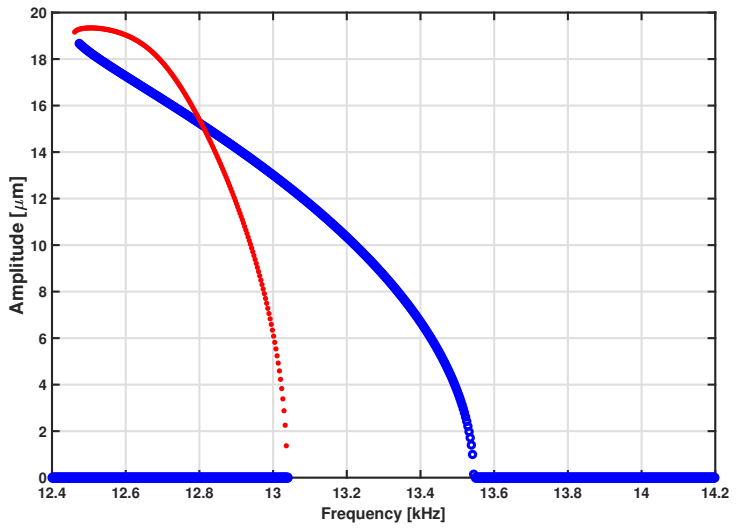

Fig. 9: Principal parametric resonance of T-beam resonator at $V_{D C}=75 \mathrm{~V}$ and $V_{A C}=30 \mathrm{~V}$. Quality factor is set to 500 .

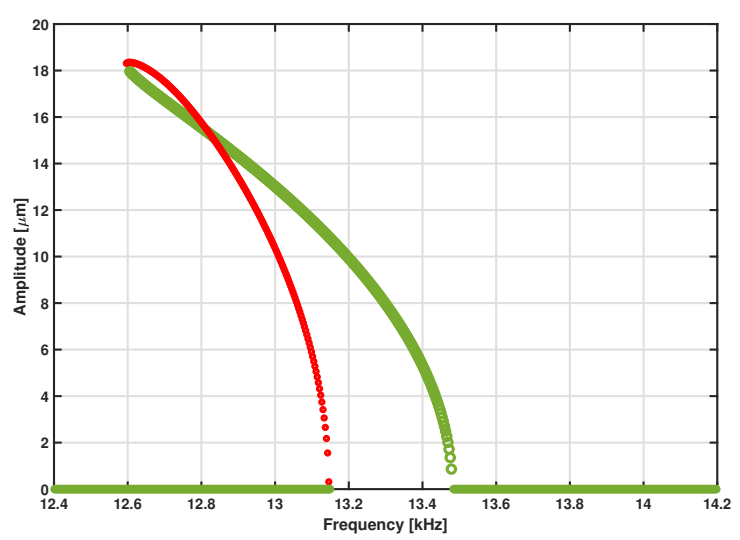

Fig. 10: Principal parametric resonance of T-beam resonator at $V_{D C}=75 \mathrm{~V}$ and $V_{A C}=20 \mathrm{~V}$. Quality factor is set to 500 .

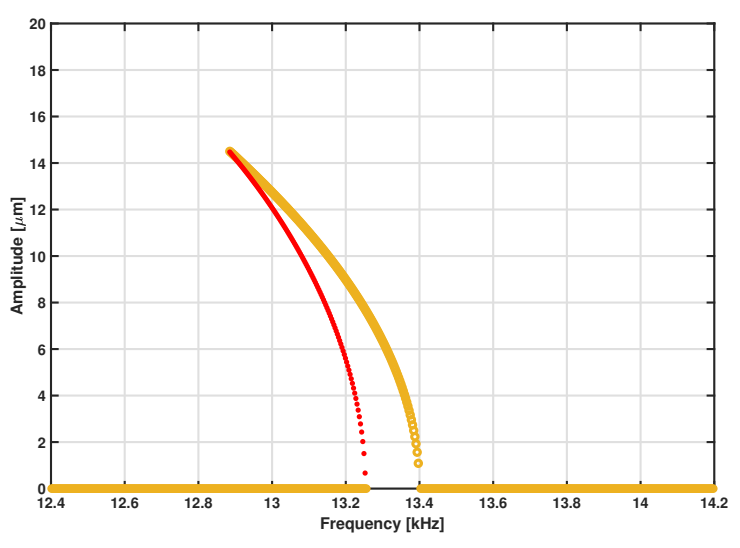

Fig. 11: Principal parametric resonance of T-beam resonator at $V_{D C}=75 \mathrm{~V}$ and $V_{A C}=10 \mathrm{~V}$. Quality factor is set to 500 . 


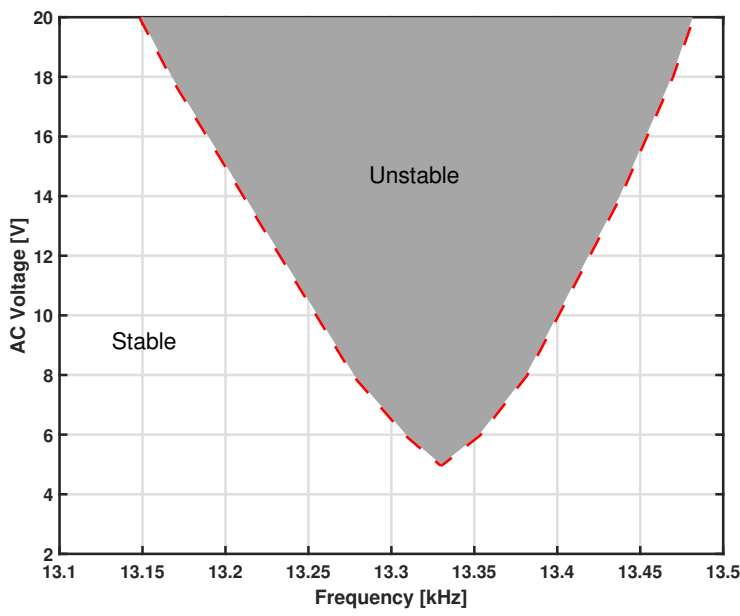

Fig. 12: Instability tongue for principal parametric resonance of T-beam resonator at $V_{D C}=75 \mathrm{~V}$. Quality factor is set to 500 .

old voltage of about $V_{A C}=4.8 \mathrm{~V}$ when quality factor is 500. The T-beam's ability to achieve unprecedented amplitudes is highly advantageous for resonance sensors where a high signal-to-noise ratio is desired. The DC voltage governs the center frequency, which provides tunability for the proposed system without pull-in instability. This is in contrast to parallel-plate devices that the increase of $\mathrm{DC}$ voltage makes the system prone to pull-in collapse.

\section{Conclusion}

A MEMS mass sensor prototype of an electrostatic micro T-beam under the influence of a repulsive force is presented in this paper. The proposed model for approximation uses an Euler-Bernoulli beam with a rigid paddle attached to the tip. Analytical and numerical responses were obtained. These results show that it is possible to predict the upper bound of the primary and parametric resonances using a one-mode approximation. The comparison made with cantilever beam under same conditions shows better properties, including higher amplitude in oscillation of primary and principal parametric resonance, which contributes to high signalto-noise ratios and enhanced resolutions. We have also presented the instability tongue to specify the region where the system shows distinct large parametric resonances. The presented T-beam resonator without pullin instability can improve the next generations of gas sensors and optical resonators to have higher sensitivity and resolution.

\section{Acknowledgment}

The authors would like to acknowledge the financial support of this study by National Science Foundation (NSF) through grant CMMI 1919608.

\section{Compliance with ethical standards}

Conflict of interest The authors declare that they have no conflict of interest.

\section{References}

1. M.I. Younis, MEMS Linear and Nonlinear Statics and Dynamics (Springer, New York, 2011)

2. S. Rahmanian, S. Hosseini-Hashemi, M. Rezaei, International Journal of Mechanical Sciences 190, 106022 (2020). DOI 10.1016/j.ijmecsci.2020.106022

3. W. Babatain, S. Bhattacharjee, A.M. Hussain, M.M. Hussain, ACS Applied Electronic Materials 3(2), 504 (2021)

4. R.N. Miles, IEEE Sensors Journal 18(14), 5691 (2018)

5. W. Zhang, H. Yan, Z. Peng, G. Meng, Sensors and Actuators A: Physical 214, 187-218 (2014). DOI 10.1016/j.sna.2014.04.025

6. S. He, R. Mrad, IEEE Transactions on Industrial Electronics 52(4) (2005)

7. K. Lee, Y.H. Cho, Microelectromechanical Systems, Journal of 10, 128 (2001). DOI 10.1109/84.911101

8. M. Pallay, M. Daeichin, S. Towfighian, Nonlinear Dynamics 89(2) (2017)

9. S. He, R. Mrad, Journal of Microelectromechanical Systems 17(3) (2008)

10. M. Ozdogan, M. Daeichin, A. Ramini, S. Towfighian, Sensors and Actuators A Physical 265 (2017). DOI 10.1016/j.sna.2017.07.043

11. M. Ozdogan, S. Towfighian, R.N. Miles, IEEE Sensors Journal 20(12), 6314 (2020)

12. M. Daeichin, M. Ozdogan, S. Towfighian, R. Miles, Sensors and Actuators A: Physical 289, 34 (2019)

13. M. Pallay, R. Miles, S. Towfighian, IEEE Transactions on Industrial Electronics (2019). DOI 10.1109/TIE.2019.2956377

14. F.N.S.C..E.M.A.R. A. H. Nayfeh, H. M. Ouakad, Nonlinear Dynamics 59, 607 (2010)

15. N. Jaber, S. Ilyas, O. Shekhah, M. Eddaoudi, M.I. Younis, in 2018 IEEE SENSORS (2018), pp. 1-4

16. N. Jaber, S. Ilyas, O. Shekhah, M. Eddaoudi, M. Younis, Sensors and Actuators A: Physical 283 (2018). DOI 10.1016/j.sna.2018.10.004

17. J. Henriksson, L.G. Villanueva, J. Brugger, Nanoscale 4 5059 (2012)

18. F. Battiston, J.P. Ramseyer, H. Lang, M. Baller, C. Gerber, J. Gimzewski, E. Meyer, H.J. Èntherodt, Sensors and Actuators B: Chemical 77 (2001). DOI 10.1016/S09254005(01)00683-9

19. A. Bouchaala, N. Jaber, O. Shekhah, V. Chernikova, M. Eddaoudi, M. Younis, Applied Physics Letters 109, 013502 (2016). DOI 10.1063/1.4955309

20. A.Z. Hajjaj, N. Jaber, N. Alcheikh, M.I. Younis, IEEE Sensors Journal 20(4), 1778 (2020) 
21. S. Krylov, R. Maimon, ASME. J. Vib. Acoust 126(3), 332-342 (2004)

22. M. Daeichin, R.N. Miles, S. Towfighian, Journal of Vibration and Acoustics (2020)

23. M. Daeichin, R.N. Miles, S. Towfighian, Nonlinear Dynamics (2020)

24. T. Rabenimanana, V. Walter, N. Kacem, P. Le Moal, G. Bourbon, J. Lardies, Sensors and Actuators A: Physical 295, 643 (2019)

25. M. Pallay, M. Daeichin, S. Towfighian, Nonlinear Dynamics 89(2), 1525 (2017)

26. M. Pallay, S. Towfighian, Sensors and Actuators A: Physical 277, 134 (2018) 
Figures

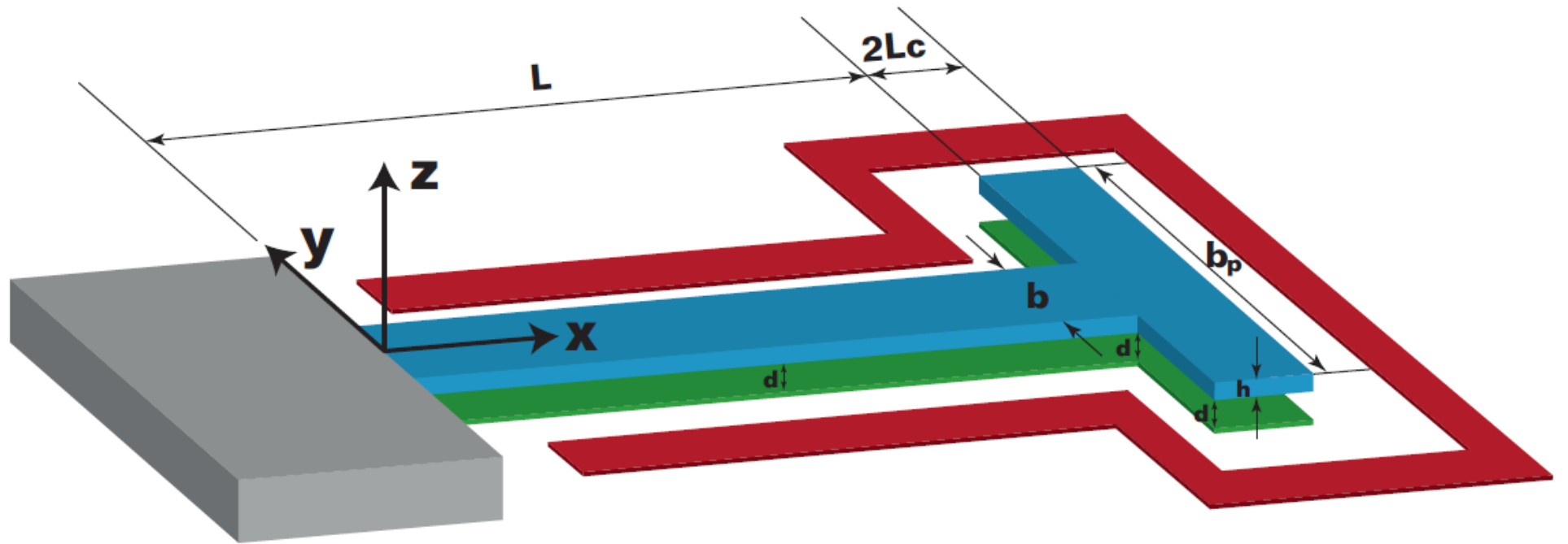

Figure 1

Schematic of the T-beam repulsive force actuator assembly.

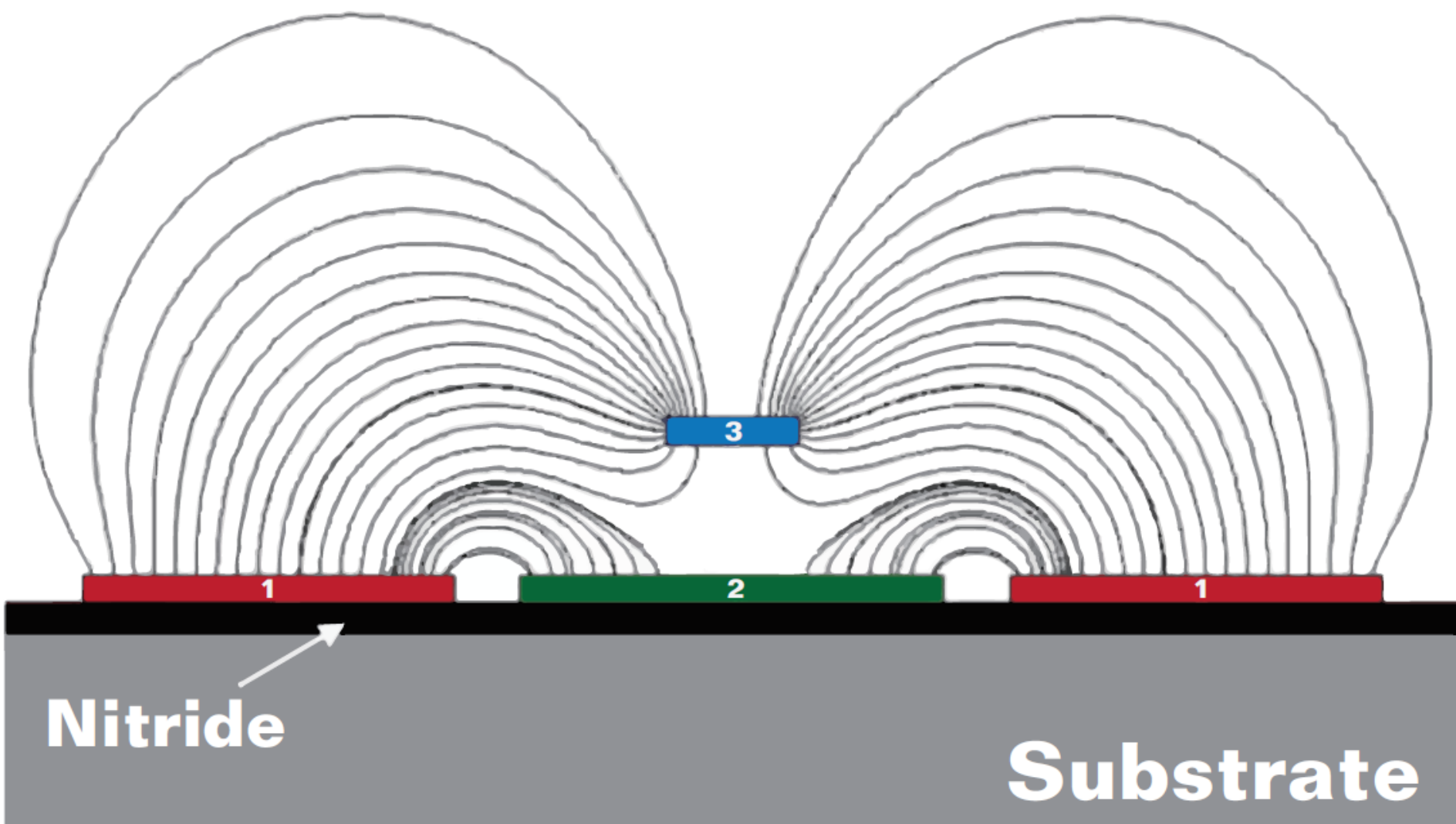

Figure 2 
Repulsive force electrode configuration with T-beam (3), fixed center electrode (2), and fixed side electrodes (1).

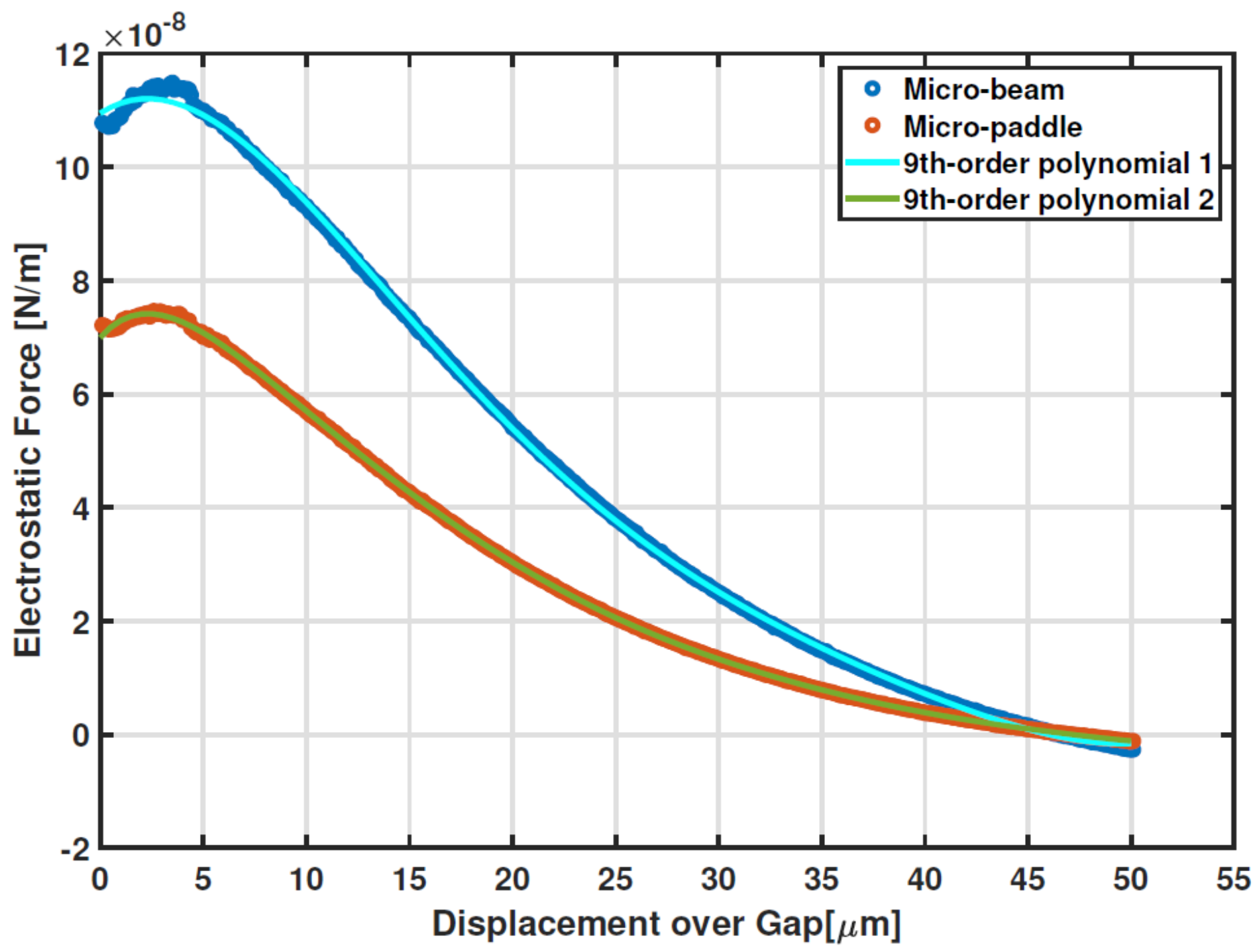

Figure 3

Electrostatic force profile for T-beam actuator shown in Figure 1. Equilibrium position at 46 microns for dimensions in Table 1. 


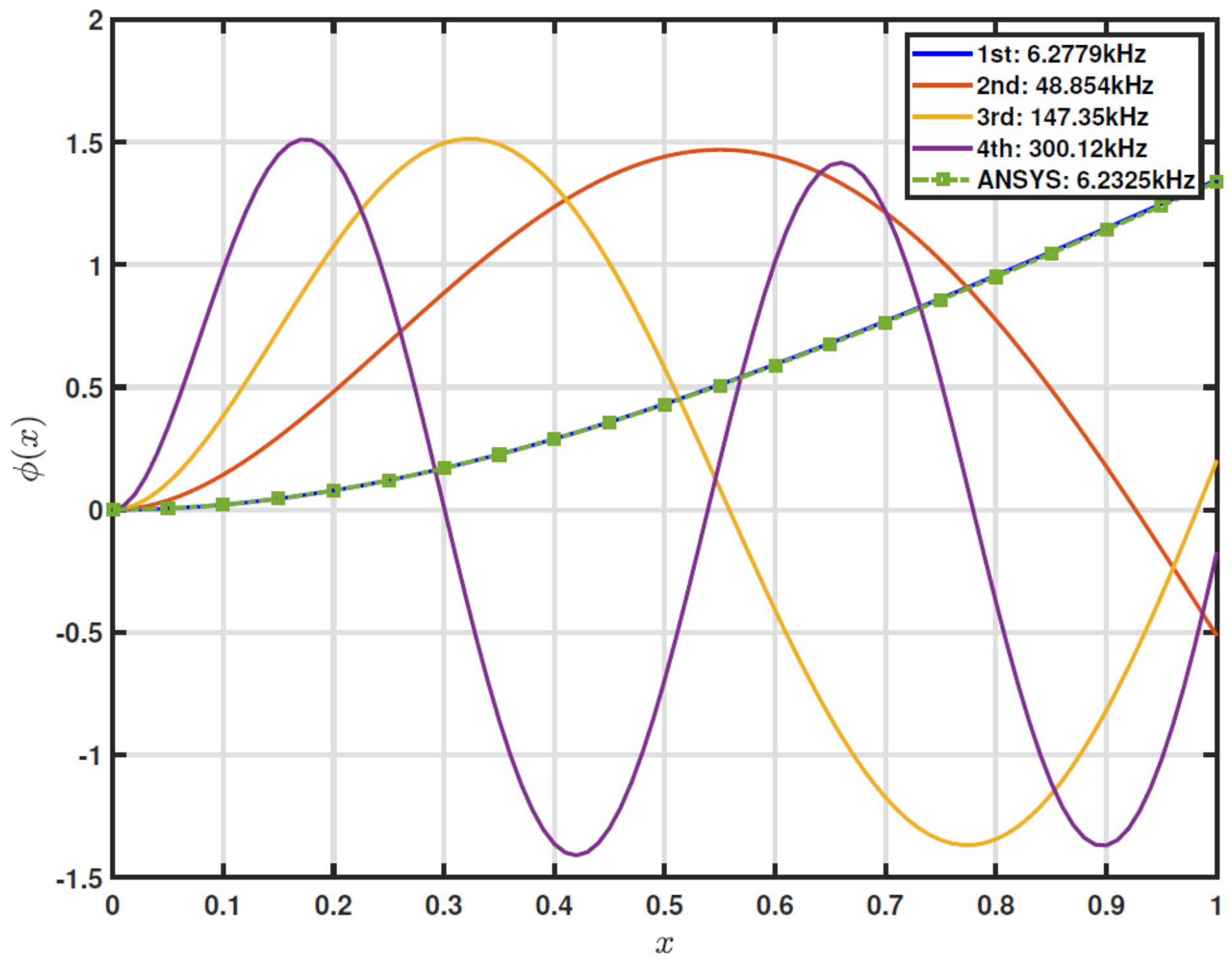

Figure 4

First four mode shapes from Matlab and first mode shape from ANSYS. 


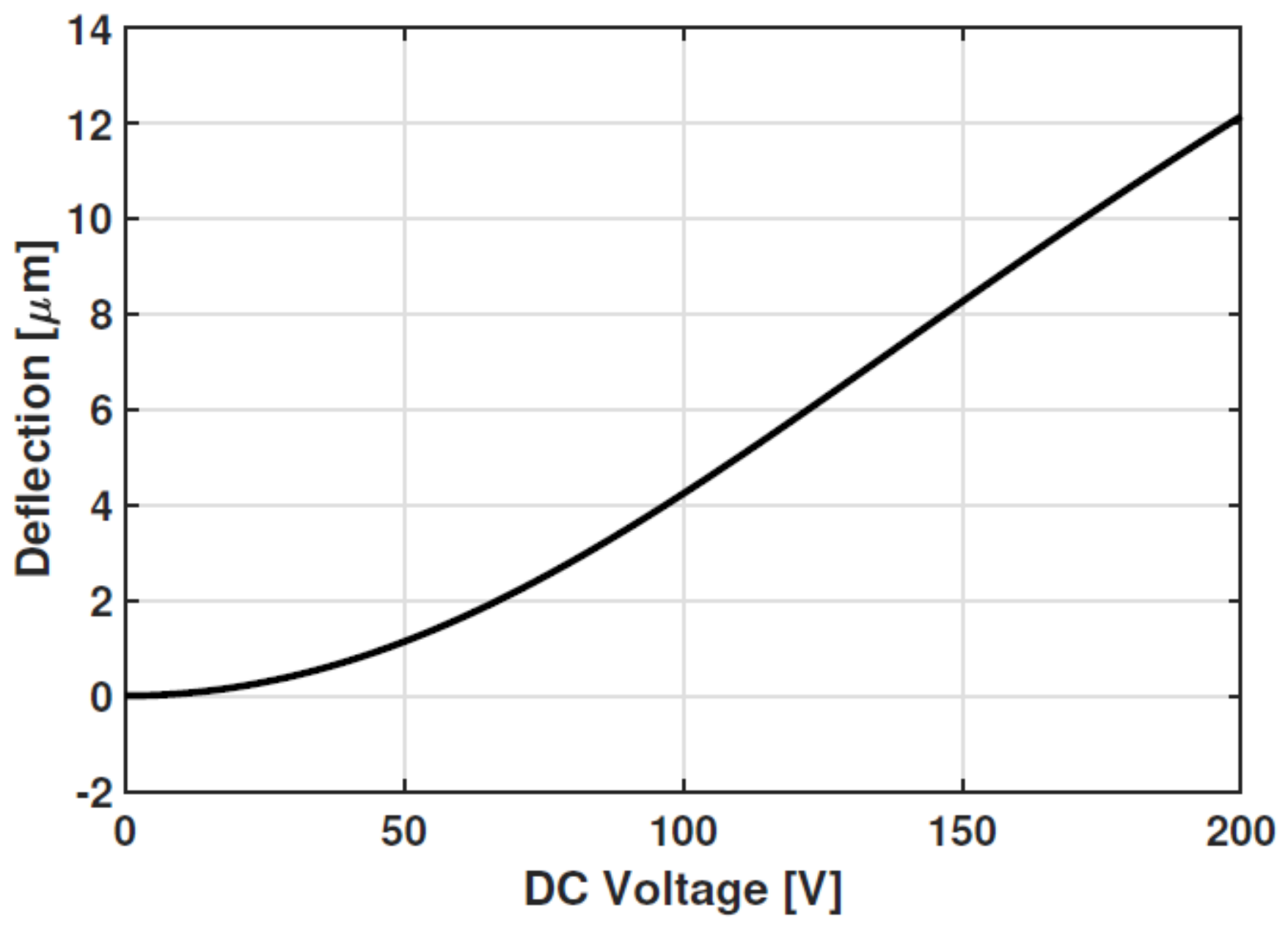

Figure 5

Static equilibrium position of the end of primary beam as the DC voltage varies. 


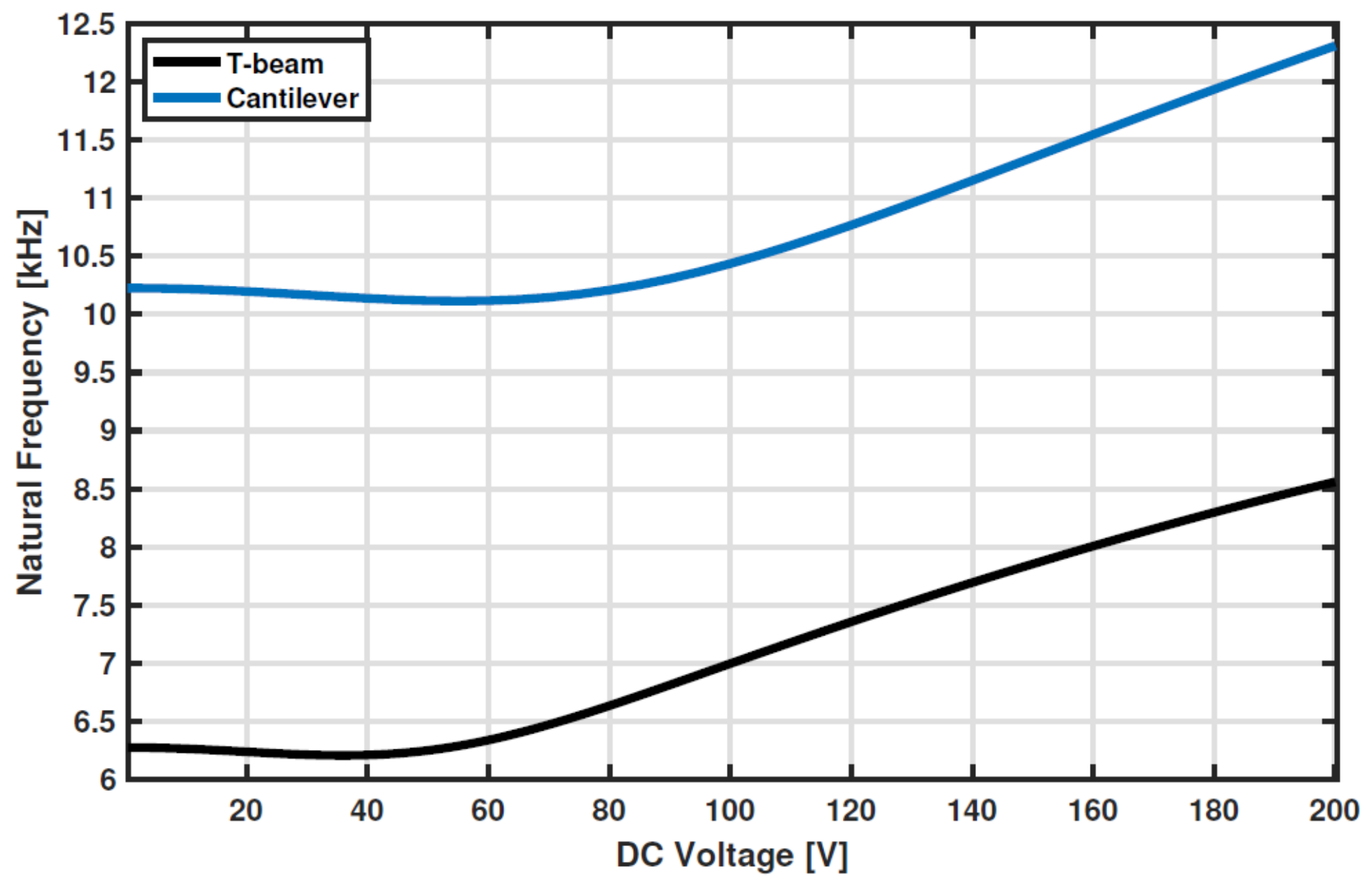

Figure 6

First natural frequency of the beam as the $\mathrm{DC}$ voltage varies. 


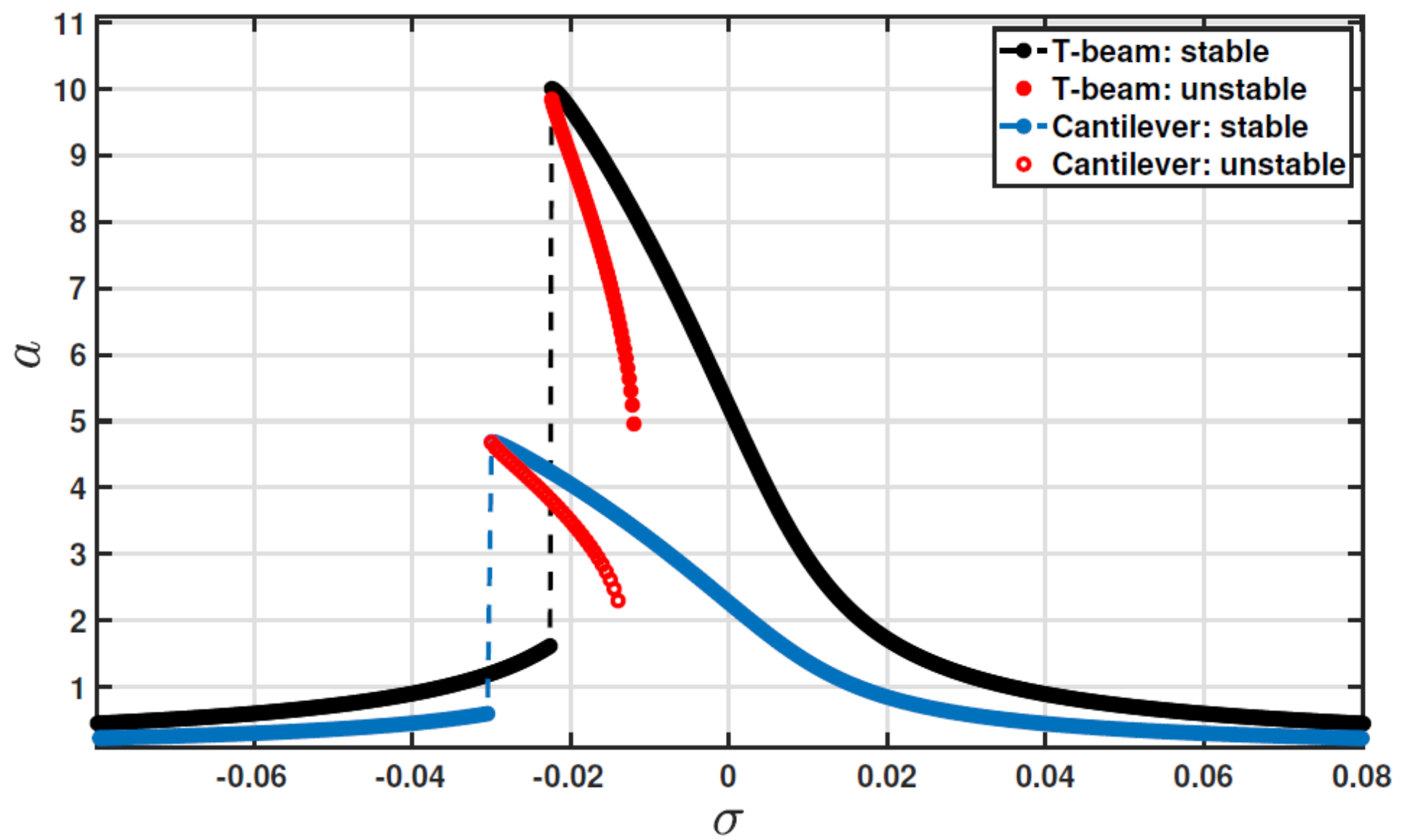

Figure 7

Comparison of primary resonant response at VDC $=195 \mathrm{~V}$ and VAC $=1 \mathrm{~V}$ for T-beam and cantilever. Quality factor is set to 130 . 


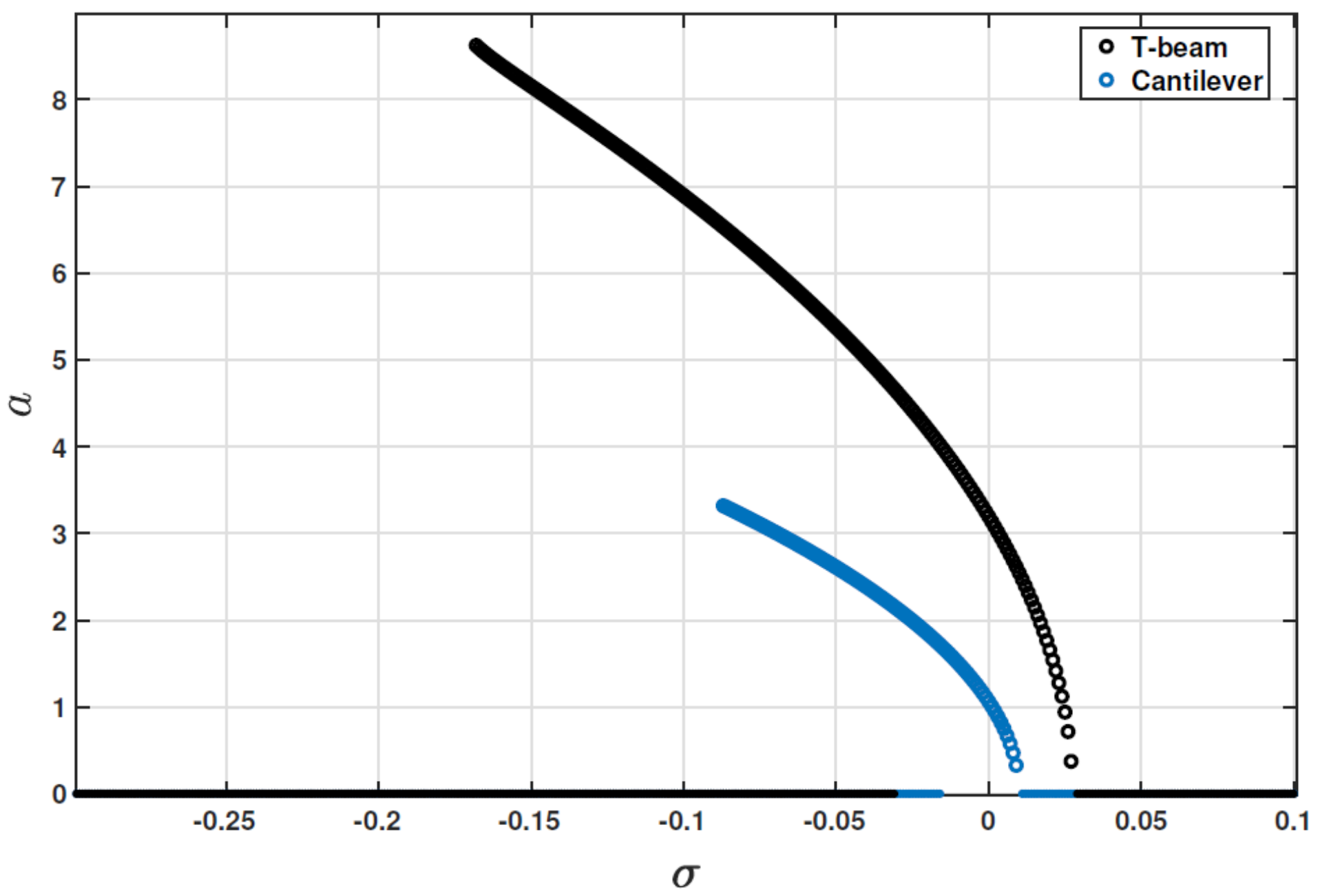

Figure 8

Comparison of principal parametric resonant response at VDC $=75 \mathrm{~V}$ and VAC $=20 \mathrm{~V}$ for T-beam and cantilever. Quality factor is set to 500 . 


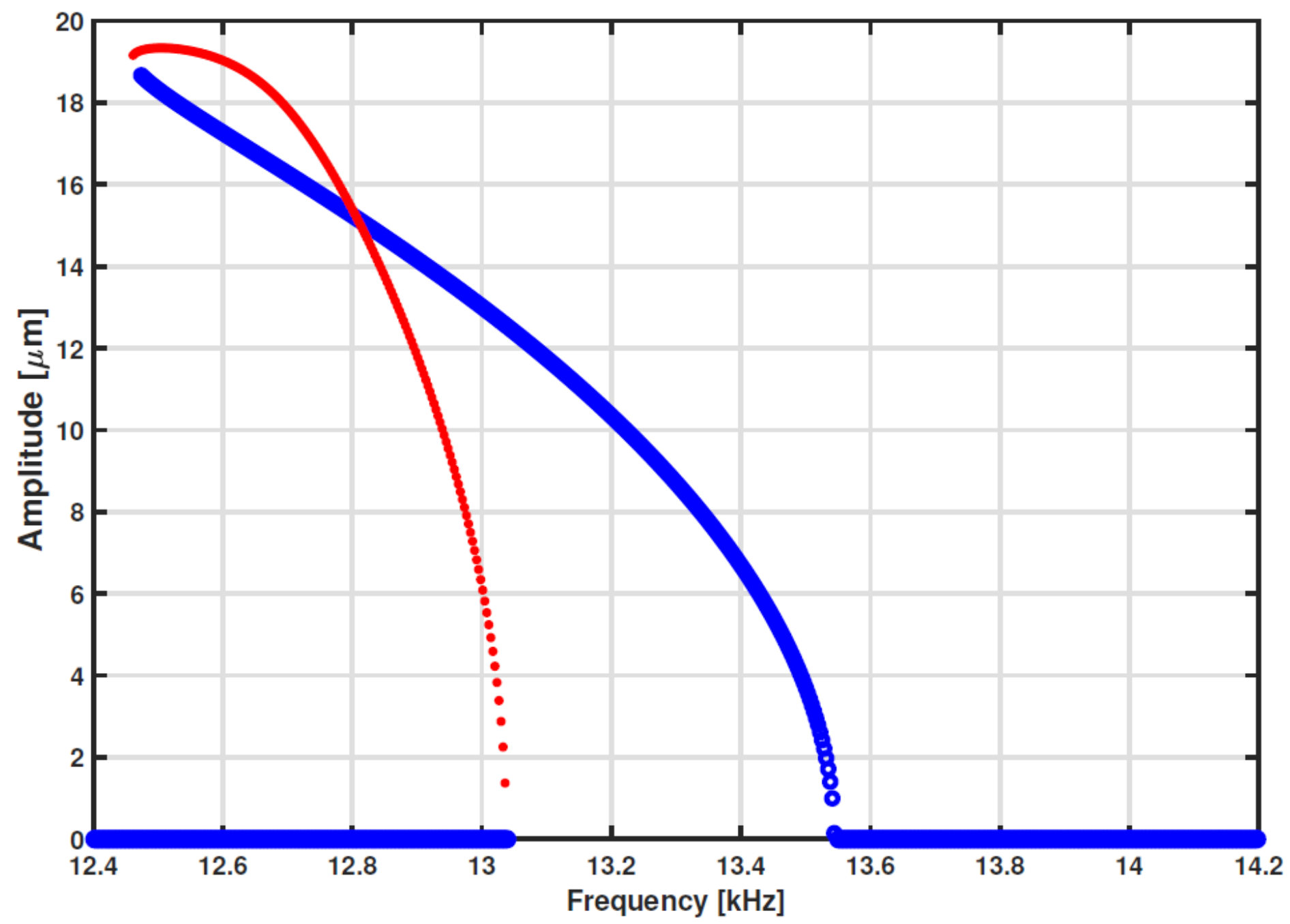

Figure 9

Principal parametric resonance of T-beam resonator at VDC $=75 \mathrm{~V}$ and $\mathrm{VAC}=30 \mathrm{~V}$. Quality factor is set to 500. 


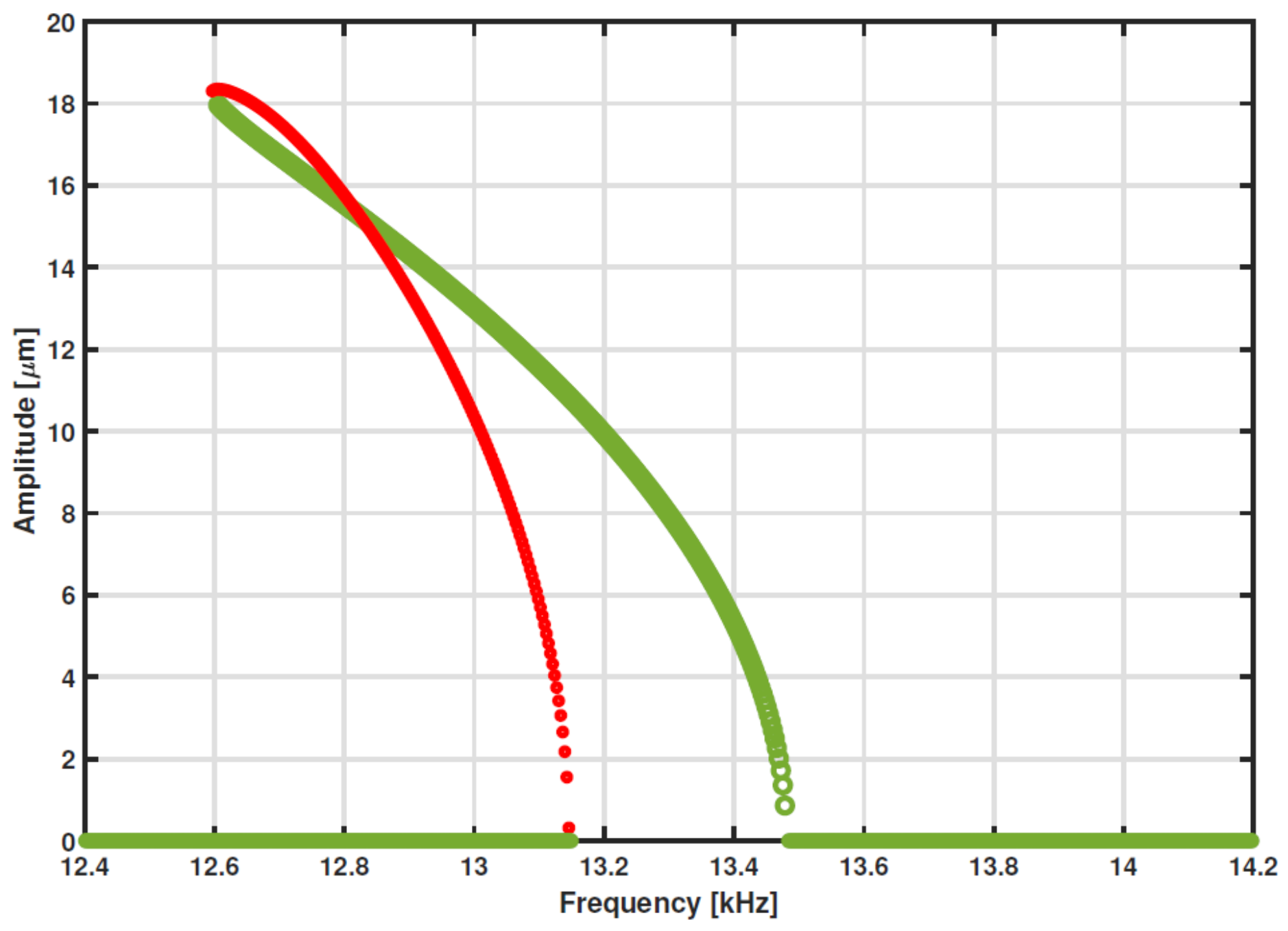

Figure 10

Principal parametric resonance of T-beam resonator at VDC $=75 \mathrm{~V}$ and $\mathrm{VAC}=20 \mathrm{~V}$. Quality factor is set to 500. 


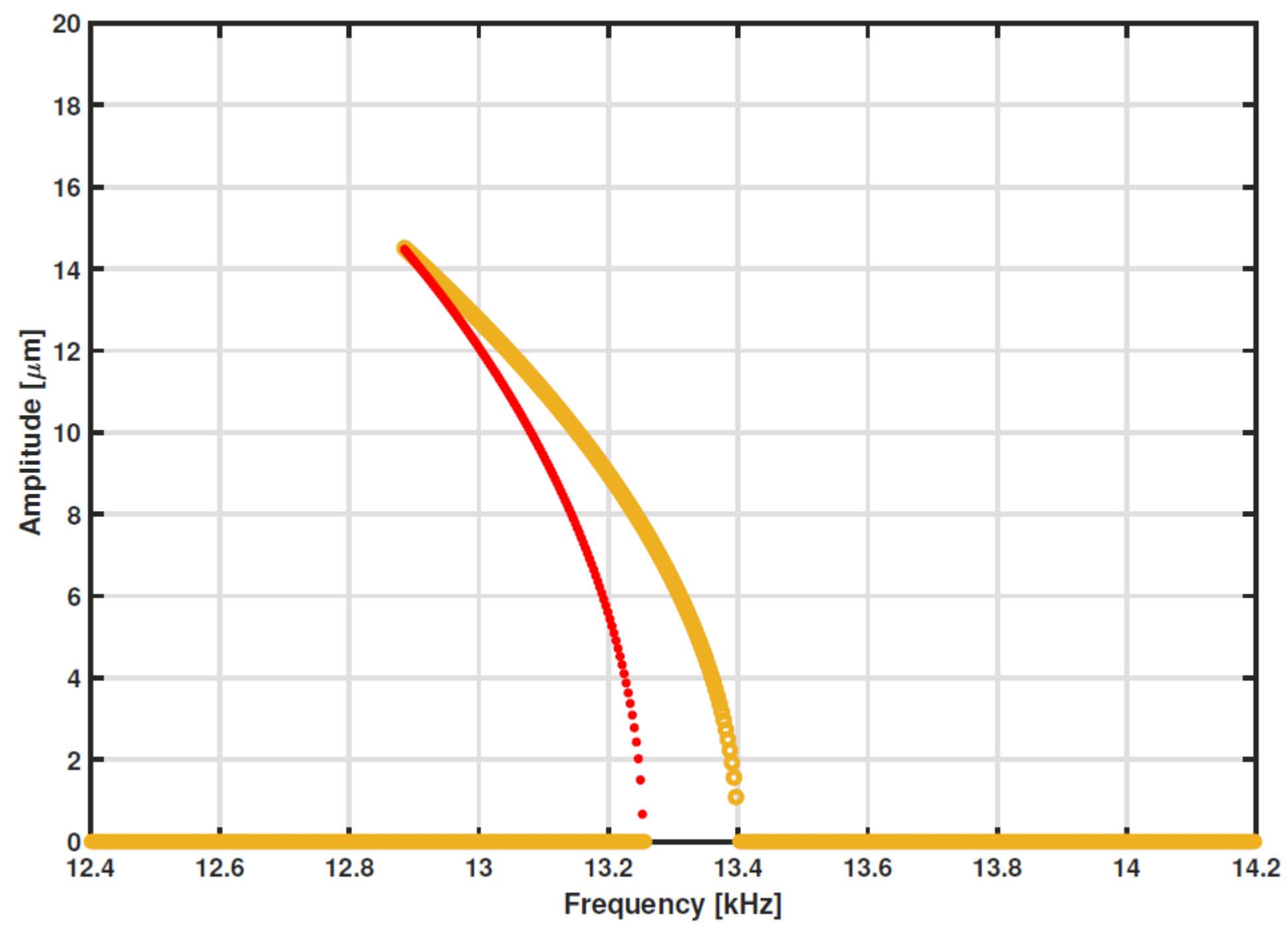

Figure 11

Principal parametric resonance of T-beam resonator at VDC $=75 \mathrm{~V}$ and $\mathrm{VAC}=10 \mathrm{~V}$. Quality factor is set to 500. 


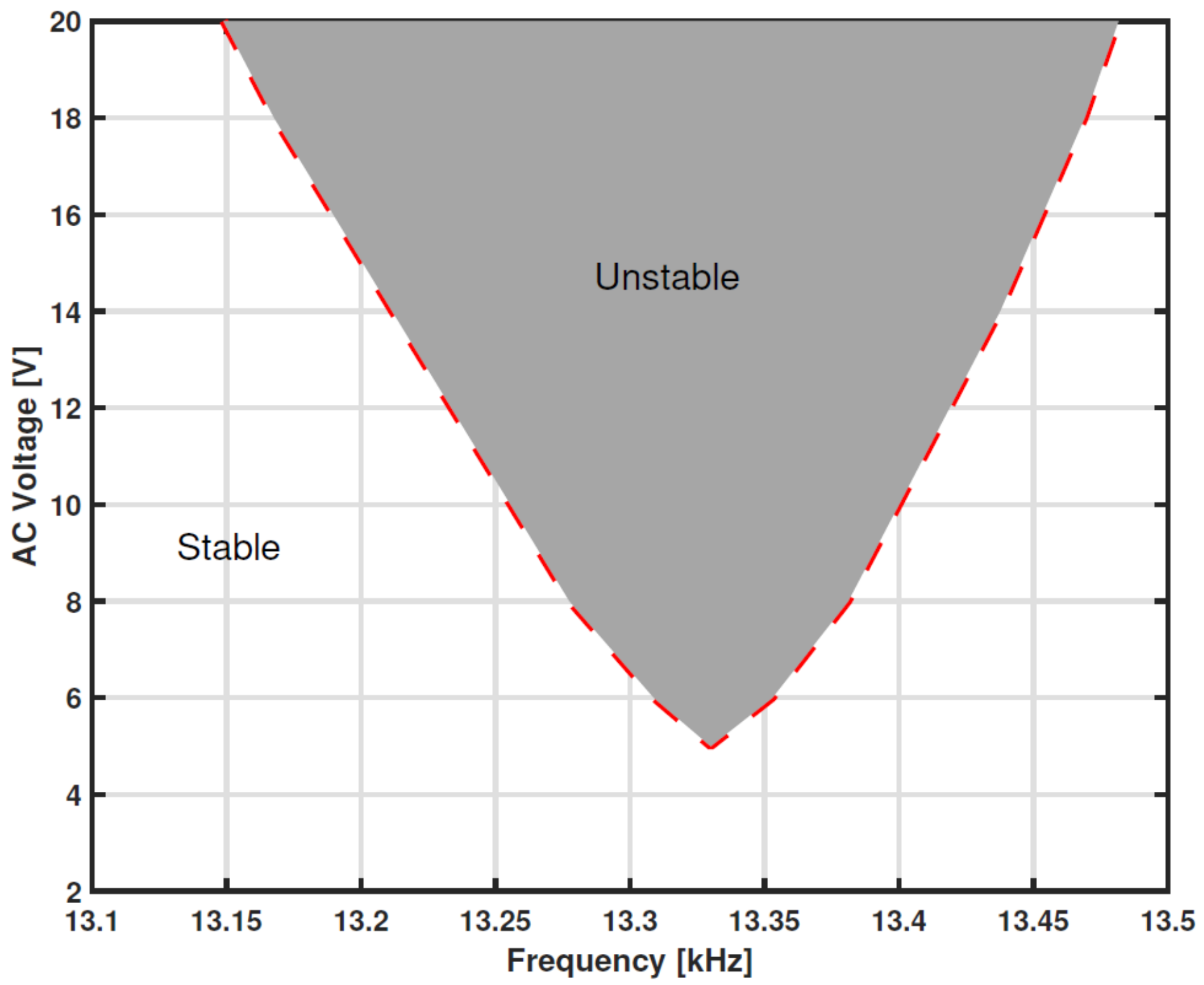

Figure 12

Instability tongue for principal parametric resonance of T-beam resonator at VDC $=75 \mathrm{~V}$. Quality factor is set to 500 . 